\title{
Involvement of Taste Receptors in the Oro-Sensory Perception of Nutrients in Rainbow Trout (Oncorhynchus mykiss) Fed Diets with Different Fatty Acid Profiles
}

\author{
Jérôme Roy (D, , ${ }^{1}$ Elodie Baranek, ${ }^{1}$ Yvan Mercier, ${ }^{1}$ Laurence Larroquet, ${ }^{1}$ Anne Surget, ${ }^{1}$ \\ Akintha Ganot, ${ }^{1}$ Franck Sandres, ${ }^{1}$ Anthony Lanuque, ${ }^{1}$ Frederic Terrier, ${ }^{1}$ and Loic Briand $\oplus^{2}$ \\ ${ }^{1}$ INRAE, Université de Pau et Des Pays de l'Adour, E2S UPPA, UMR1419 Nutrition Metabolism and Aquaculture, Aquapôle, \\ F-64310 Saint-Pée-Sur-Nivelle, France \\ ${ }^{2}$ Centre Des Sciences du Goût et de l'Alimentation, AgroSup Dijon, CNRS, INRAE, Université Bourgogne Franche-Comté, \\ Dijon, France
}

Correspondence should be addressed to Jérôme Roy; jerome.roy@inrae.fr

Received 11 October 2021; Revised 13 December 2021; Accepted 18 December 2021; Published 31 January 2022

Academic Editor: Zhen-Yu Du

Copyright (c) 2022 Jérôme Roy et al. This is an open access article distributed under the Creative Commons Attribution License, which permits unrestricted use, distribution, and reproduction in any medium, provided the original work is properly cited.

\begin{abstract}
Taste perception plays an important role in an animal's detection of nutrients, conveying key dietary information, fundamental for its growth and survival. Because alternative terrestrial ingredients are known to affect the feeding of rainbow trout (RT, Oncorhynchus mykiss), we aimed to assess the importance of taste receptors in detection. Using self-feeders, we examined the feeding behavior (30 days of a feeding trial followed by 10 days of a preference trial) of RT fed with a commercial diet (C), vegetable diets supplemented with linseed oil (V1) or algal oil (V2). During the feeding trial those fed V2 decreased their food intake. The preference trial revealed that fish preferred V2 $v$. C and V1 v. V2 for fish which had consumed V1 and C during their feeding trial. Mechanistically, taste receptors were mainly expressed in taste organs and regulated by diet, which indicated the function of the taste receptors. Some taste receptors for fatty acids (such as the ffar receptor) and amino acids (such as the tasr receptor) were highly expressed in the RT tongue. While ffar2a transcripts were upregulated by vegetal diets in the tongue, ffar1 and ffar4, known for important roles in mammals, were very low expressed and not found in the RT genome, respectively. Overall findings show that RT displayed the fundamental mechanisms for oro-gustatory perception of nutrients related to different diet composition.
\end{abstract}

\section{Introduction}

Over the past 20 years, to cope with the booming growth and the environmental and ecological impacts of aquaculture, feeding protocols of farmed fishes have had to be adapted. Availability of traditional aquafeed ingredients, including fish meal (FM) and fish oil (FO), has not increased with demand, and today the traditional ingredients of aquafeeds must be replaced by renewable, eco-friendly and economically viable alternative products [1]. Carnivorous fish species, such as salmonids, are among the highest consumers of FO and FM. Among salmonids, carnivorous rainbow trout (RT, Oncorhynchus mykiss) farming is the main freshwater producer in Europe and intense research efforts are being focused on replacing marine ingredients (FM and FO) with more easily available plant-based ingredients in their diets. However, after 20 years of research [2], the total replacement of marine products by plant ingredients for RT production has still not been achieved and several bottlenecks remain. Previous studies revealed that the total replacement of FM and FO with plant ingredients from first feeding of RT led to reduction of growth and survival rates [3], mainly related to altered feeding behavior.

Moreover, another disadvantage of using plant ingredients in fish farming production is the modification of the nutrient composition of the diet. By far, the major difference in terms of nutrient replacement in plant-based diets $v$. commercial diets (containing FM/FO) is the lack of essential $\omega$-3 
long chain polyunsaturated fatty acids ( $\omega-3$ LC-PUFA), mainly eicosapentaenoic acid (EPA) and docosahexaenoic acid (DHA) [4]. Consequently, even if RT has the capacity to biosynthesize $\omega$-3 LC-PUFA [5] from their precursor alpha-linolenic acid (ALA), this synthesis is too low compared to direct nutritional intake, resulting in a huge reduction in the DHA and EPA flesh content [6].

Some studies reported that this absence of $\omega$-3 LC-PUFA in plant products, especially DHA, is known to affect feeding behavior during the whole fish life-cycle by reducing food intake (FI) [7], swimming activities, and feeding rhythms [8], and by increasing abnormal feeding behavior [9]. Recently, we highlighted that $\omega$-3 LC-PUFA controlled the feeding behavior of juvenile RT [10]. Under voluntary FI, we reported that fish could distinguish between diets containing different levels of $\omega$-3 LC-PUFA and exhibit a preference for diets containing high levels of EPA and DHA. Furthermore, we observed that a diet rich in $\omega$-3 LC-PUFA accounted for a relatively high proportion of brain function [11] and brain lipid content [12] in RT juveniles. These recent studies have disclosed implications of $\omega$-3 LC-PUFA on the modulation and regulation on the control of FI particularly the fatty acid (FA) sensing pathways even though molecular pathways remain to be elucidated.

Taste is the main driver of food approach and choice, fundamental for diet selection in vertebrates [13]. This is the first system involved in sensory detection of nutrients that play a key role in the regulation of feeding behavior and energy balance [14]. Prior to the involvement of the central nervous system [15], the perception and discrimination of thousands of different palatable nutrients by the vertebrate's gustatory systems originate from the activation of specific taste receptors. Taste receptors including several classes of G protein-coupled receptors (GPCR) recognize nutrients and respond by inducing intracellular calcium signals. These trigger the release of bioactive compounds and influence other organs. These gustatory receptors have been shown to be expressed in numerous extra-oral tissues and organs. Among numerous functions [13], taste receptors help to coordinate the response to the meal, and contribute to the regulation of satiation [16]. In mammals, even dietary fat detection has traditionally been considered to rely moreon texture and olfaction rather than considered as a sense of taste. Mounting evidence supports the notion that oral fat also activates the gustatory system [17-19]. In particular, in mammals numerous membrane proteins expressed in the taste receptor cells are essential for fat detection including the free fatty acid receptor family (FFAR) such as FFAR1 and FFAR4 $[20,21]$ or a cluster of differentiation 36 (CD36) [22]. However, research for the oro-detection of long-chain fatty acids have been mainly conducted on rodent models as well as on human taste bud cells [23] but remains poorly studied in fishes [24]. Hovewer, the description of the morphological characteristics of the tongue of RT has recently been published [25]. The study revealed orogustatory structures common to mammals (the presence of taste bud cells), but the molecular mechanism underlying the lingual detection of nutrients has not been elucidated in fishes.
In this study, we aimed to characterize and identify for the first time in farmed fish the oro-sensory detection operating in the tongue by the detection of taste receptors for nutrients. Among taste receptors, the present study focused on receptors that can act as a sensor with selectivity for the long-chain fatty acids [26] (the free fatty acid receptor family; FFAR and GPR84, GPR119, or CD36). We also focused on fatty acid receptors known to be expressed in RT [27] but never studied for oro-sensory detection (GPR84, GPR119). Although different fatty acids in the diets were the major focus of this study, we could not exclude a modulation of other taste receptors known to act as sensors for other nutrients in mammals, especially because they have never been studied in RT. Thus, other receptors including the taste receptor 1 family (T1R), the calcium-sensing receptor (casr), and GPR92/93, known to be involved in amino acid sensing [28] were studied.

The study aimed to characterize the distribution of taste receptors in the buccal cavity (tongue, palace, pharynx and lips), and also in tissues known to express taste receptors in mammals [29] and fishes (gill filament, liver, intestine and stomach) [30]. Secondly, we deciphered the modulation of taste receptors in the tongue (presence of taste bud cells) of RT related to different diets. For this, we compared the effects of two plant-based diets supplemented with or without Schizochytrium sp. micro-algae oil (enriched in DHA) with those of a commercial diet (containing FM/FO rich in DHA) on feeding activity or food preference in juvenile RT. Using a self-feeding system, we investigated first the feeding behavior of juvenile RT fed with one of these diets. A second trial observed the adaptation of RT to an environmental change by eating and choosing an alternative diet and noting the influence of a previous diet (diet programming during the first trial). This information on the detection and regulation of taste receptors is important in aquaculture in the diversification or substitution of feed ingredients, especially from the already expensive and limited FM and other animal-based ingredients.

\section{Materials and Methods}

2.1. Ethics Statement. The experiment was conducted following the Guidelines of the National Legislation on Animal Care of the French Ministry of Research (Decree No 2013118, 1 February 2013) and in accordance with EU legal frameworks relating to the protection of animals used for scientific purposes (i.e. Directive 2010/63/EU). The scientists in charge of the experiments received training and personal authorization. The experiment was conducted at the INRAE NuMeA facilities (permit number A64.495.1 delivered by French veterinary services), and approved by the ethical committee (C2EA-73) of INRAE "Comité d'éthique Aquitain poissons oiseaux" ( $\mathrm{N}^{\circ}$ agreement INRAE 21699, $19^{\text {th }}$ December, 2019). All efforts were made to minimise the number of fish used and their suffering. No clinical symptoms were observed within or outside the experimental periods.

2.2. Experimental Diets. Diets were manufactured at the INRAE experimental facilities at Donzacq using a twinscrew 
extruder (Clextral). Details about the ingredients and composition of the experimental diets are given in Table 1, FA proportions in the diets in Table 2 and amino acid proportions in the diet (estimated) in supplemental information 2.

The experiment was conducted with three different experimental diets: a commercial-like diet (C) containing a mix of FM (27\%), FO (12.1\%) and plant ingredients, and two total plant-based diet (V1 and V2), completely free from FM and FO, which were replaced by a blend of plant ingredients. In order to have the closest proportions of $\omega-3 \mathrm{FA}$ classes found in marine and plant diets [3]. The three experimental diets contained $21.17 \%$ crude lipids. For the sake of clarity in the feeding behavior analysis and to conclude if $\omega$ 3 LC-PUFA affected modulation of the sensory gustatory perception, we made the choice to vary only one $\omega$-3 LCPUFA in each experimental diet. Thus, the three experimental diets contained the same amount of one major $\omega-3 \mathrm{FA}$; $26.52 \%$ of DHA in C diet, $27.62 \%$ of ALA in V1 diet and $26.25 \%$ of DHA in V2 diet.

To obtain this amount of DHA in the C diet (representing $77 \%$ of $\omega$-3 LC-PUFA) and to avoid a minimal contamination by EPA (only $11 \%$ of $\omega$-3 LC-PUFA in the C diet), a marine oil concentrated in DHA [Omegavie ${ }^{\circledR}$ DHA oil (min $70 \%)$ ] was added (7.3\%) with other FO (4.8\%). V1 and V2 diets were formulated based on the same feed ingredient composition, differing only in their vegetable oil derivation (Table 1). This blend was chosen in order to provide an overall amount of FA (Table 2) and amino acid classes (supplemental information 2) and was very similar to the proportion of FA classes found in $\mathrm{C}$ diet and only varying by one $\omega$-3 LC-PUFA. For the V1 diet, DHA (present in FO for the $\mathrm{C}$ diet) was replaced to increase the amount of alpha-linolenic acid (ALA) by adding linseed oil (10\%).

For V2 diet, to observe if the absence of DHA in the vegetable diet could affect the modulation of sensory gustatory perception of RT, the dietary content of DHA was increased by adding a vegetable oil [Omegavie ${ }^{\circledR}$ DHA 700 algae sensor oils (Polaris functional lipids, Quimper, France)]. This vegetable oil was used to substitute linseed oil in order to replace ALA in the V1 diet.

To avoid exceeding anti-nutrient threshold levels, we used a blend of wheat gluten, extruded peas and whole wheat, corn gluten meal, rapeseed meal and white lupin as protein sources (c. $44.36 \%$ of the total diet). Synthetic Llysine, L-methionine, dicalciumphosphate and soy-lecithin were added to each diet to correct the deficiency in essential amino acids, phosphorous and phospholipids. Mineral and vitamin premix were added to each diet. Diets have all been formulated to be iso-proteic, iso-lipidic, and iso-energetic and to cover the nutritional requirements of RT (according to [31]).

2.3. Proximate Composition. Nutrient compositions of the diets were analyzed by drying the samples to constant weight at $105^{\circ} \mathrm{C}$ for $24 \mathrm{~h}$. Crude protein was determined by the Kjeldahl method after acid digestion and the concentration was estimated by multiplying the nitrogen content using the 6.25 factor as commonly used [32]. Crude lipids were quantified by the Soxhlet method using petroleum diethyl ether for the extraction as previously described [12]. Gross energy was determined with an adiabatic bomb calorimeter (IKA, Heitersheim Gribheimer, Germany). Starch content was evaluated by an enzymatic method (Megazyme). Ash content was determined by combustion in a muffle furnace $\left(550^{\circ} \mathrm{C}\right.$ for $\left.8 \mathrm{~h}\right)$. Total lipid was extracted and measured gravimetrically according to the Folch method [33] using dichloromethane instead of chloroform. FA methyl esters were prepared by acid-catalyzed transmethylation of total lipids using boron trifluoride $\left(\mathrm{BF}_{3}\right)$ in methanol $(14 \%)$ according to the Shantha and Ackman method [34] and analyzed in a Varian 3900 gas chromatograph (Varian, les Ulis, France) equipped with a fused silica DB wax capillary column $(30 \mathrm{~m} \times 0.25 \mathrm{~mm}$ internal diameter, film thickness $0.25 \mu \mathrm{m}$; JW Alltech, France). Injection volume was $1 \mu \mathrm{l}$, using helium as a carrier gas $(1 \mathrm{ml} / \mathrm{min})$. The temperatures of the injector and the flame ionization detector were 260 and $250^{\circ} \mathrm{C}$, respectively. The thermal gradient was as follows: $100-180^{\circ} \mathrm{C}$ at $8^{\circ} \mathrm{C} / \mathrm{min}, 180-220^{\circ} \mathrm{C}$ at $4^{\circ} \mathrm{C} / \mathrm{min}$ and a constant temperature of $220^{\circ} \mathrm{C}$ for $20 \mathrm{~min}$. FA were identified with reference to a known standard mixture (Sigma, St Louis, MO, USA) and peaks were integrated using Varian Star Chromatography Software (Star Software, version 5). Individual FAs were expressed as a percentage of the total FAME identified.

2.4. Rearing and Self-Feeders. Female RT used in this experiment originated from the same parental stock (INRAE Fish Farm of Lees-Athas, Permit number A64.104.1, vallée d'Aspe, France). The feeding trial was conducted in a recirculating rearing system at the INRAE facilities of SaintPee-sur-Nivelle, France (Permit number A64.104.1). Details about experimental procedure are summarized in supplemental information 1.

Before starting the experiment, fish (mean 65) were reared and acclimatized to the experimental conditions (tank and self-feeder) and fed a commercial feed (Neostart1, Le Gouessant aquaculture, Lamballe, France) for 3 weeks (by hand twice a day for the first week and by self-feeders for the second 2 weeks). After the acclimation period, juveniles were randomly distributed among 15 fiberglass tanks (Table 3). Between 16 to 17 fish were randomly distributed per tank $(79.75 \pm 0.49 \mathrm{~g})$ in stocking density at the beginning of the trial $\left(c .13 \mathrm{~kg} / \mathrm{m}^{3}\right.$ per tank). Although the initial number of fish per tank was higher than necessary to be statistically reliable for the analysis, this initial of juvenile RT was essential for a successful feeding behavior experiment. Compared to the mammalian model in research, salmonids such as RT form linear, dominance-based, social hierarchies in both natural and artificial populations in aquaculture systems [35]. These social hierarchies depending on stocking densities are essentials throughout the life cycle of RT impacting their growth [36], feeding rhythm (feed intake and conversion efficiency of the fish) [37], survival [38] and physiological status [39].

Tanks of $100 \mathrm{~L}$ were used and water flow was set to ensure an oxygen concentration $>90 \%$ saturation. The tanks were individually aerated, and re-circulated water was thermostatically maintained at $17.2 \pm 0.2^{\circ} \mathrm{C}$ (flow rate, $31 \mathrm{~min}^{-1}$ ). Dissolved 
TABLE 1: Ingredients and composition of the experimental diets.

\begin{tabular}{|c|c|c|c|}
\hline Ingredient (g/100 g dry weight) & Commercial-like & $\begin{array}{l}\text { DIET } \\
\text { Vegetal V1 }\end{array}$ & Vegetal V2 \\
\hline Fish meal & 27.0 & 0.0 & 0.0 \\
\hline Extruded whole wheat & 22.5 & 17.0 & 17.0 \\
\hline Corn gluten & 8.7 & 17.0 & 17.0 \\
\hline Wheat gluten & 7.0 & 17.0 & 17.0 \\
\hline Peas meal & 3.0 & 6.0 & 6.0 \\
\hline Extruded peas & 7.0 & 9.5 & 9.5 \\
\hline White lupin seed meal & 2.5 & 4.5 & 4.5 \\
\hline Rapeseed meal & 0.8 & 3.0 & 3.0 \\
\hline Soy lecithin & 0.0 & 2.0 & 2.0 \\
\hline L-lysine & 0.5 & 1.7 & 1.7 \\
\hline L-methionine & 0.0 & 0.4 & 0.4 \\
\hline $\mathrm{CaHPO}_{4} \cdot 2 \mathrm{H}_{2} \mathrm{O}$ & 0.0 & 1.4 & 1.4 \\
\hline Mineral premix ${ }^{\mathrm{a}}$ & 1.0 & 1.0 & 1.0 \\
\hline Vitamin premix ${ }^{\mathrm{b}}$ & 1.0 & 1.0 & 1.0 \\
\hline Fish oil & 4.8 & 0.0 & 0.0 \\
\hline Palm oil & 2.0 & 5.0 & 6.0 \\
\hline Sunflower oil & 4.9 & 2.7 & 4.7 \\
\hline Linseed oil & 0.0 & 10.8 & 0.0 \\
\hline DHA marine oil (from Polaris) ${ }^{c}$ & 7.3 & 0.0 & 0.0 \\
\hline DHA 700 algae sensor (from Polaris) ${ }^{\mathrm{d}}$ & 0.0 & 0.0 & 7.75 \\
\hline \multicolumn{4}{|l|}{ Composition (estimated) } \\
\hline Crude protein & 48.00 & 48.00 & 48.00 \\
\hline Crude lipid & 21.80 & 21.90 & 21.80 \\
\hline Starch & 20.00 & 20.00 & 20.00 \\
\hline Energy (kJg-1 DM) & 24.12 & 24.44 & 24.84 \\
\hline
\end{tabular}

${ }^{a}$ Mineral premix: (g or mg kg- 1 diet): calcium carbonate ( $\left.40 \% \mathrm{Ca}\right), 2.15 \mathrm{~g}$; magnesium oxide (60\% Mg), $1.24 \mathrm{~g}$; ferric citrate, $0.2 \mathrm{~g}$; potassium iodide (75\% I), $0.4 \mathrm{mg}$; zinc sulphate ( $36 \% \mathrm{Zn}), 0.4 \mathrm{~g}$; copper sulphate $(25 \% \mathrm{Cu}), 0.3 \mathrm{~g}$; manganese sulphate $(33 \% \mathrm{Mn}), 0.3 \mathrm{~g}$; dibasic calcium phosphate ( $20 \% \mathrm{Ca}, 18 \% \mathrm{P}), 5 \mathrm{~g}$; cobalt sulphate, $2 \mathrm{mg}$; sodium selenite ( $30 \% \mathrm{Se}$ ), $3 \mathrm{mg}$; KCl, $0.9 \mathrm{~g}$; NaCl, $0.4 \mathrm{~g}$ (UPAE, INRA). ${ }^{\mathrm{b}}$ Vitamin premix: (IU or mg kg- 1 diet): DL-a tocopherol acetate, $60 \mathrm{IU}$; sodium menadione bisulphate, $5 \mathrm{mg}$; retinyl acetate, 15,000 IU; DL-cholecalciferol, $3000 \mathrm{IU}$; thiamin, $15 \mathrm{mg}$; riboflavin, $30 \mathrm{mg}$; pyridoxine, $15 \mathrm{mg}$; B12, $0.05 \mathrm{mg}$; nicotinic acid, $175 \mathrm{mg}$; folic acid, $500 \mathrm{mg}$; inositol, $1000 \mathrm{mg}$; biotin, $2.5 \mathrm{mg}$; calcium pantothenate, $50 \mathrm{mg}$; choline chloride, $2000 \mathrm{mg}$ (UPAE, INRA). ${ }^{\circ}$ Omegavie ${ }^{\circledR}$ DHA oil (min 70\%): concentrated marine oil produced mainly from anchovy and sardine oil. The crude oil is first refined, then purified and concentrated. This oil contains marine DHA Omega 3 fatty acids under Triglycerides form. From POLARIS, Quimper, France. ${ }^{\mathrm{d}}$ Omegavie ${ }^{\circledR}$ DHA 700 algae sensor oil (min 70\%): Oil from the micro-algae Schizochytrium sp., natural mixed tocopherols E306, vegetable oil (MCT), sunflower lecithin E322, ascorbyl palmitate E304, rosemary extract E392. This oil contains marine DHA Omega 3 fatty acids under Triglycerides form. From POLARIS, Quimper, France.

oxygen $\left(\mathrm{mgl}^{-1}\right), \mathrm{pH}(7.5)$ and water temperature were continuously monitored via probes. $\mathrm{NH}_{4}^{+}\left(0.0-0.1 \mathrm{mgl}^{-1}\right)$, $\mathrm{NO}_{2}{ }^{-}$(0.025-0.100 $\mathrm{mgl}^{-1}$ ) (Microquant test kit for $\mathrm{NH}_{4}$ and $\mathrm{NO}_{2}$; Merck KGaA, Darmstadt, Germany) were measured daily.

Each tank was equipped with two self-feeding devices (Imetronic ${ }^{\circledR}$, Pessac, France) positioned at opposite sides of the tank. The trigger consisted of a simple $10 \mathrm{~cm}$ long and $0.4 \mathrm{~cm}$ thick stainless steel rod that could pivot freely around its axis. When the rod moved, a proximity switch positioned at the apex closed a logical $5 \mathrm{~V}$ electric circuit. The entire system was connected to a computer that compiled and recorded the time, the date and the tank and feeders from which each feed demand and distribution originated [40]. Control software recorded the origin of the signals and controlled the feeders. The detectors and the feeders were interfaced directly with a simple electronic device that triggered the feeder when the rod was struck by a fish. The computer delivered a predetermined amount of food (c. 45 pellets, $2.5 \mathrm{~g}$ ) which was calculated by regularly weighing the feed remaining in the feed hoppers during the acclimation period.

Every day, the measurement of waste (uneaten pellets) that accumulated in the sediment trap in the water outlet of each tank was noted to check how much of the distributed feed had been consumed. Each trigger was also placed $1 \mathrm{~cm}$ above the water surface to avoid unintentional trigger activations by the fish. As the fish had unrestricted feed distribution during the feeding period (each demand was eligible for a food reward), a delay of $1 \mathrm{~s}$ between two feed rewards was established to ensure that each demand was an intentional FI. This precautionary measure prevented multiple activation per demand due to the sensitivity of the actuation. 
TABLE 2: Selected fatty acid composition (\% total fatty acids).

\begin{tabular}{|c|c|c|c|}
\hline & \multicolumn{3}{|c|}{ Diet } \\
\hline & $\begin{array}{c}\text { Commercial- } \\
\text { like }\end{array}$ & $\begin{array}{l}\text { Vegetal } \\
\text { V1 }\end{array}$ & $\begin{array}{l}\text { Vegetal } \\
\text { V2 }\end{array}$ \\
\hline C12:0 & 0.10 & 0.18 & 0.22 \\
\hline C14:0 & 1.70 & 0.33 & 0.49 \\
\hline $\mathrm{C} 15: 0$ & 0.3 & 0.04 & 0.04 \\
\hline C16:0 & 12.20 & 15.37 & 15.02 \\
\hline $\mathrm{C} 17: 0$ & 0.2 & 0.07 & 0.05 \\
\hline C18:0 & 2.84 & 3.67 & 2.38 \\
\hline C20:0 & 0.31 & 0.24 & 0.23 \\
\hline $\mathrm{C} 22: 0$ & 0.30 & 0.24 & 0.30 \\
\hline $\mathrm{C} 24: 0$ & 0.10 & 0.14 & 0.13 \\
\hline $\begin{array}{l}\text { Sum of saturated fatty } \\
\text { acids }\end{array}$ & 18.07 & 20.2 & 18.84 \\
\hline $\mathrm{C} 14: 1 \omega-7$ & 0.05 & 0.0 & 0.02 \\
\hline $\mathrm{C} 16: 1 \omega-7$ & 2.07 & 0.17 & 0.25 \\
\hline C18:1 $\omega-9$ & 16.72 & 24.74 & 19.33 \\
\hline C20:1 $\omega-9$ & 1.69 & 0.24 & 0.18 \\
\hline $\mathrm{C} 22: 1 \omega-9$ & 1.77 & 0.0 & 0.0 \\
\hline Sum of MUFAs & 22.30 & 25.15 & 19.78 \\
\hline $\mathrm{C} 18: 2 \omega-6$ & 20.30 & 26.34 & 26.34 \\
\hline $\mathrm{C} 18: 3 \omega-6$ & 0.13 & 0.0 & 0.0 \\
\hline $\mathrm{C} 20: 2 \omega-6$ & 0.17 & 0.0 & 0.0 \\
\hline $\mathrm{C} 20: 3 \omega-6$ & 0.11 & 0.0 & 0.05 \\
\hline $\mathrm{C} 22: 2 \omega-6$ & 0.05 & 0.10 & 0.09 \\
\hline $\mathrm{C} 22: 4 \omega-6$ & 0.32 & 0.0 & 0.0 \\
\hline $\mathrm{C} 22: 5 \omega-6$ & 1.56 & 0.0 & 4.94 \\
\hline Sum of $\omega-6$ LC-PUFAs & 24.09 & 26.61 & 31.56 \\
\hline $\mathrm{C} 16: 4 \omega-3$ & 0.04 & 0.06 & 0.03 \\
\hline C18:3 $\omega-3$ (ALA) & 0.80 & 27.62 & 1.14 \\
\hline $\mathrm{C} 18: 4 \omega-3$ & 0.43 & 0.0 & 0.0 \\
\hline $\mathrm{C} 20: 3 \omega-3$ & 0.12 & 0.0 & 0.0 \\
\hline $\mathrm{C} 20: 4 \omega-3$ & 0.34 & 0.0 & 0.27 \\
\hline $\mathrm{C} 20: 5 \omega-3$ (EPA) & 4.08 & 0.0 & 0.91 \\
\hline $\mathrm{C} 21: 5 \omega-3$ & 0.24 & 0.0 & 0.0 \\
\hline $\mathrm{C} 22: 4 \omega-3$ & 0.14 & 0.0 & 0.0 \\
\hline $\mathrm{C} 22: 5 \omega-3$ & 1.96 & 0.0 & 0.14 \\
\hline $\mathrm{C} 22: 6 \omega-3$ (DHA) & 26.52 & 0.19 & 27.25 \\
\hline Sum of $\omega-3$ LC-PUFAs & 34.67 & 27.86 & 29.73 \\
\hline $\begin{array}{l}\text { Sum of } \omega-3(\text { EPA } \\
+ \text { DHA })\end{array}$ & 30.6 & 0.19 & 28.16 \\
\hline$\omega-3(\mathrm{DHA}+\mathrm{EPA}) / \omega-6$ & 1.27 & 0.0 & 0.89 \\
\hline
\end{tabular}

Feed demands were rewarded during two feeding period of $2.5 \mathrm{~h}(06: 30-09: 00$ and 18:30 - 21:00 hours), corresponding to the maximal feeding periods under unrestricted conditions [40]. During the acclimation period, fish were conditioned to feed during these feeding trials by means of an extra $40 \mathrm{~W}$ light. For the artificial photoperiod (mimicking the natural photoperiod) the light was switched on at 06:00 and off at 21:00 hours, including an artificial dawn and dusk period of $30 \mathrm{~min}$ each ( $15.5 \mathrm{~h}$ light: $8.5 \mathrm{~h}$ dark). During this habituation phase, at the beginning of each feeding period (06:30 - 18:30 hours), the interface offered a reward (without fish actuation), which warned RT of the beginning of the feeding period. During the feeding period, the monitor displayed a food reward after activation by RT. After the feeding period (9:00 - 21:00 hours), RT could touch the trigger but no food reward would be provided. The conditionning period of RT to the self-feeder device was achieved in 10 days [41].To avoid oxidation of the diet and to stabilise the feed, pellets (stored in the dark and cool in an airtight bag) were regularly (every other day) added to the feeders.

2.5. Feeding Trials I and II. The experimental plan of the present study was performed in two sequential trials: a first period of feeding of 30 days and second period of preference of 10 days (supplemental information 1). At the end of the acclimation period, a 30 day feeding phase was performed. Each tank (five by diet) distributed the same diets through two self-feeders (Table 3 and supplemental information 1). For this first trial, the aim was to observe the feeding behavior (voluntary consumption and waste) of the fish between the $\mathrm{C}, \mathrm{V} 1$ and V2 experimental diets and to observe the impact of the experimental diet in the modulation of taste receptors. For this, at the end of 30 days, $6 \mathrm{~h}$ after the last meal, we selected eight RT (one or two per tank, all dominant fish easily identifiable by their lighter color compared to their congeners, $n=8$ in total per diet). This choice to sample the dominant fish ensured that the selected RT were those that had intentionally activated the triggers and thus intentionally consumed the diet by choice [38] rather than having consumed the remaining pellets of the group (which does not reflect the voluntary feeding effect of the diet). Prior to sampling, confirmation was established that the fish had consumed the feed. Fish were first anesthetized in a $30 \mathrm{mg} \mathrm{L}^{-1}$ benzocaine bath and then sacrificed in a $60 \mathrm{mg} \mathrm{L}^{-1}$ benzocaine bath for $6 \mathrm{~h}$ after the last meal. This sampling time was chosen after kinetic experiments performed in another study in our laboratory (study not published). To decipher the overall distribution and expression of taste receptors in RT, the lip, palate (hard palate, superior), pharynx, gill filament, liver, intestine, stomach and heart were dissected out in the $\mathrm{C}$ diet group. The central area of the hypothalamus (regulating food intake in fish and thus for the analysis of neuropeptides) and the proximal tongue (for diet effect) for the three experimental diets (the largest fish from the same fish used to compare diets for the $\mathrm{C}$ dietary group) were dissected out. All tissues were immediately frozen in liquid nitrogen and stored at $-80^{\circ} \mathrm{C}$ for further analysis.

After the first trial, 10 days of preference testing were performed for the remaining fish (15-16 per tank) fed for 30 days with one of the three experimental diets. The experimental set-up has already been published [42]. Compared to the first trial, each tank had two different diets delivered by the self-feeders which could be chosen by the fish. These two diets were the diets not consumed during the first trial. Preference observations in five tanks per test were made on 
TABLE 3: Mean values ( \pm SEM) of growth performance, feed parameters and digestive energy intake of rainbow trout fed with different diets for 30 days before the preference trial. Values are expressed as group mean \pm SEM; One-way ANOVA, Tukey post hoc; Different lower case letters indicate significant differences $(P<0.05)$ among diets.

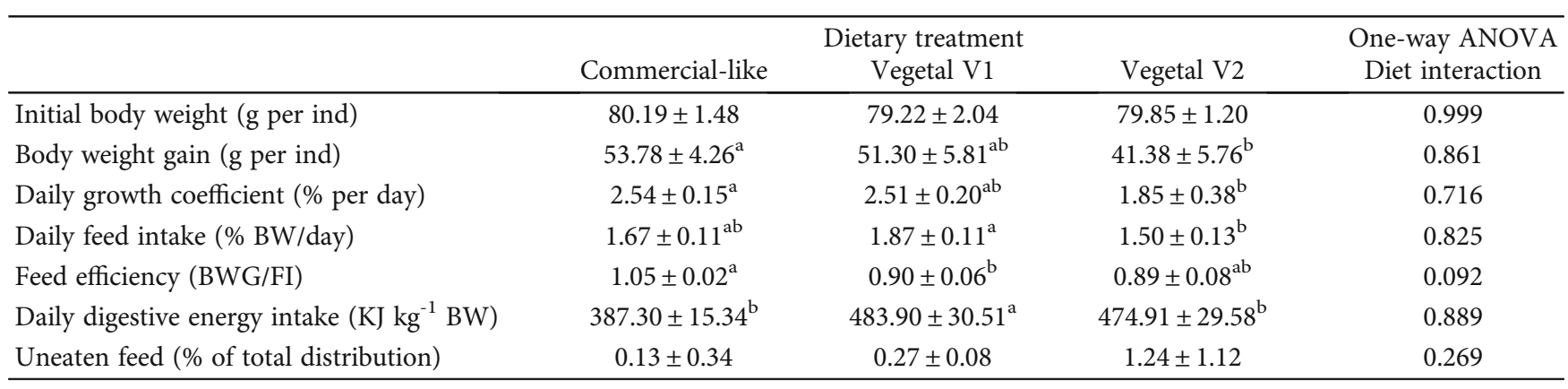

fish which had consumed the $\mathrm{C}$ diet during the feeding trial and were offered the choice between the V1 and V2 diets., Fish which had consumed the V1 diet during the feeding trial were offered the choice between the $\mathrm{C}$ and V2 diets, and fish which had consumed the V2 diet during the feeding trial were offered the choice between the $\mathrm{C}$ and V1 diets. The trial was stopped 10 days after a clear preference or no preference (not just a daily feed preference for one diet) for the proposed diets was observed. The aim of this second trial was to observe adaptation of RT to an environmental change by eating and choosing an alternative diet. It was also to observe the effect of diet conditioning (diet programming during the feeding trial) and the impact of the nutrient composition on food choices.

2.6. Variables and Analysis. Juvenile RT were counted and weighed as a group at the beginning and at the end of each trial. No intermediate weighing was done to avoid stress and loss in appetite due to handling. The number of demands and their distribution among the self-feeders, the feed remaining in the feeders and the amount of uneaten feed were recorded daily. This was done by emptying the decantation tube connected at the outlet of each tank into a sieve and weighing and counting the settled feed pellets. For this, different shades of green (using natural dyes added in the oil mixture) between pellets allowed the two diets in each tank to be discriminated. Color was chosen based on previous experiments and known not to affect feed preferences [10].

During the feeding trial, total biomass was recorded at the beginning and at the end of the trial. Variables related to the zootechnic parameters are presented in Table 3 for the feeding trial and Figures 1 and 2 for the preference trial. Variables related to growth were:

Body weight gain $(B W G, g)=$ final body weight $(F B W)$ initial body weight (IBW).

Daily growth coefficient (DGC, \% per day) $=100 x$ (FBW0.33 - IBW0.33)/days.

Daily FI (\% body weight per day)= total amount of feed consumed per day/100 $\times$ BWG per day.

Feed efficiency $=F B W-I B W / F I$.

Variables related to FI were corrected for the amount of uneaten feed and expressed in relative terms (\% of body weight, BW, per day).
Daily digestive energy intake $(D E I)=F I x$ Digestible energy (DE content) of the diet (estimated as $23.07 \mathrm{kJg}^{-1}$ ).

The variables related to feed preference during the preference trial, the daily or cumulative preferences ( $\%$ total feed distribution), were calculated as relative changes in the feed demands for one diet to the cumulative feed preference at the end of the feeding trial. As already published [10], feed preference expressed as a relative change was necessary to be sure that the diet preference observed will be related to the diet rather than a preference acquired during the feeding trial for the self-feeder position in the tank. At the end of feeding trial, feeder activity within each tank was compared to analyze feeder preferences, independently of the diet. The preferred feeder was assigned as the one receiving $>50 \%$ of total feed demands over the last 10 days of the feeding trial $\left(d_{-10}-d_{0}\right)$. The initial preference ratio $\left(P_{\text {ini }}\right)$ was calculated:

$P_{\text {ini }}=100 \times$ number of feed distribution in the preferred feeder $d_{-10^{-}} d_{0 /}$ total number of feed distributions during $d_{-10^{-}}$ $d_{0 /} 50$.

The $P_{\text {in }}$ ratio varied between 2 in the case where feeder distribution occurred only for this feeder and 0 in the case of complete avoidance of the previously preferred feeder during the feeding period. The highest $P_{\text {ini }}$ ratio caculated between two feeders in one tank was $1.56 v .0 .44$. Thus, daily or cumulative preferences during the preference trial were calculated as relative changes in the feed demands for one diet as:

Daily preference $=100 \times$ number of feed distributions for the feeder/(total number of feed distributions for the tank $X$ $P_{\text {ini }}$ for the feeder concerned).

The proportion of uneaten feed (UF) was expressed as a percentage of total feed distributed during the feeding trial (Table 3) and total pellet weight (g) for the preference trial (Figure 2(c)). Feed parameters were analyzed over the total first feeding trial and preference trial.

2.7. Gene Expression Analysis: Quantitative Real-Time PCR. Total RNA was extracted from the tongue and hypothalamus for the three dietary groups and from the lip, palate (hard palate, superior), pharynx, gill filament, liver, intestine, stomach and heart for the $\mathrm{C}$ dietary group ( $n=6$ per diet) using the TRIzol reagent method (Invitrogen, Carlsbad, CA) with Precellys ${ }^{\circledR} 24$ (Bertin technologies, Montigny le 


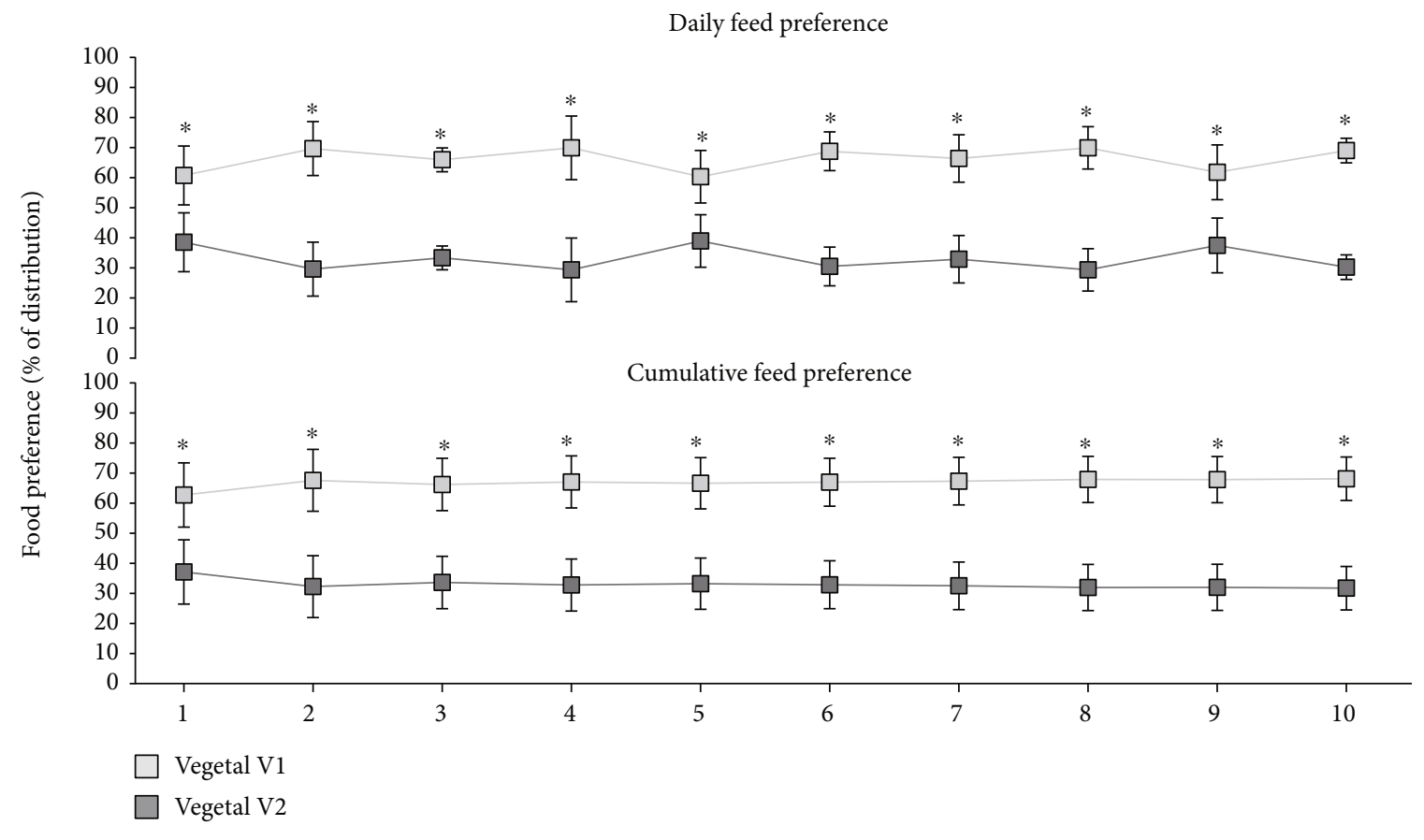

(a)

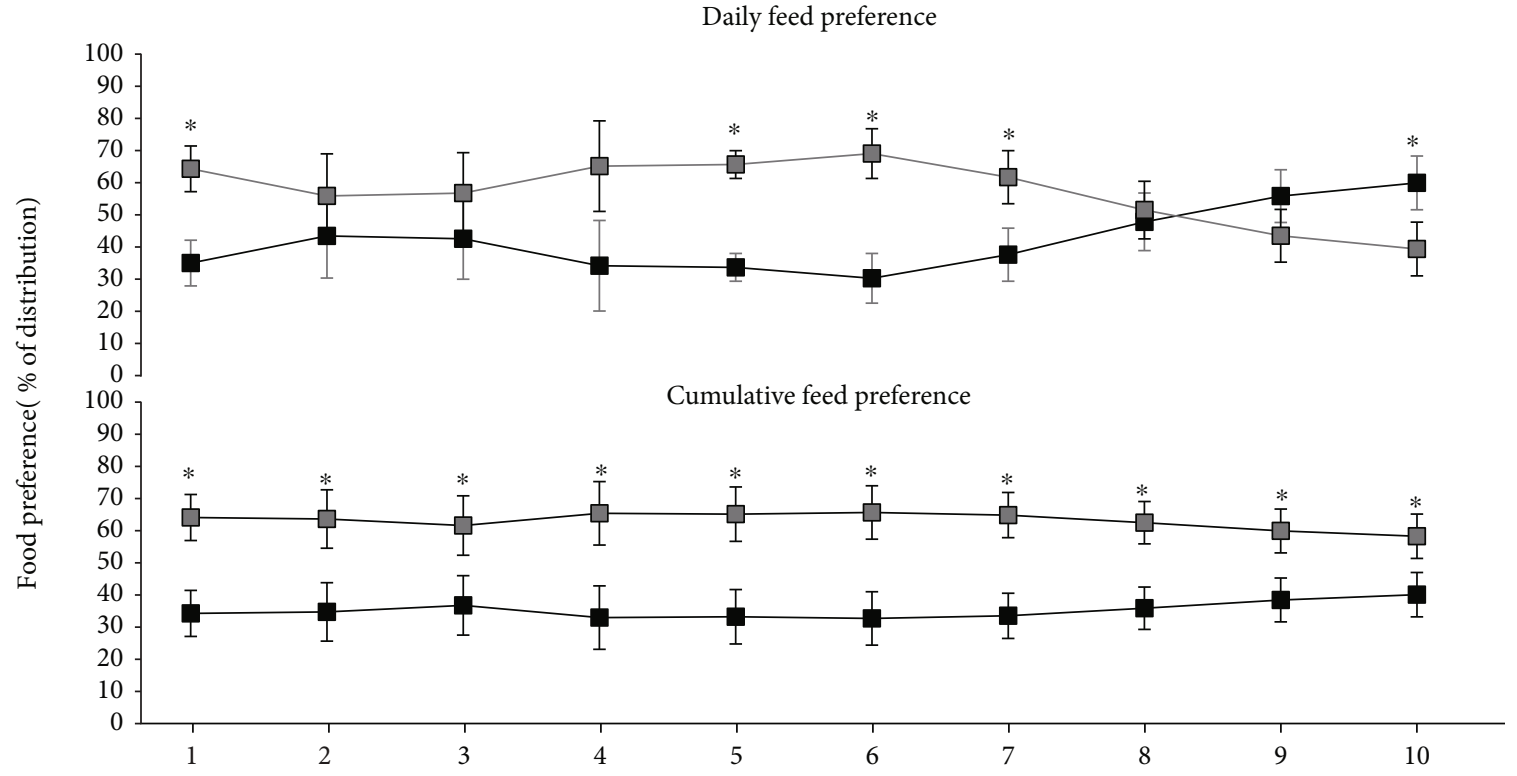

Vegetal V2

Commercial-like

(b)

Figure 1: Continued. 


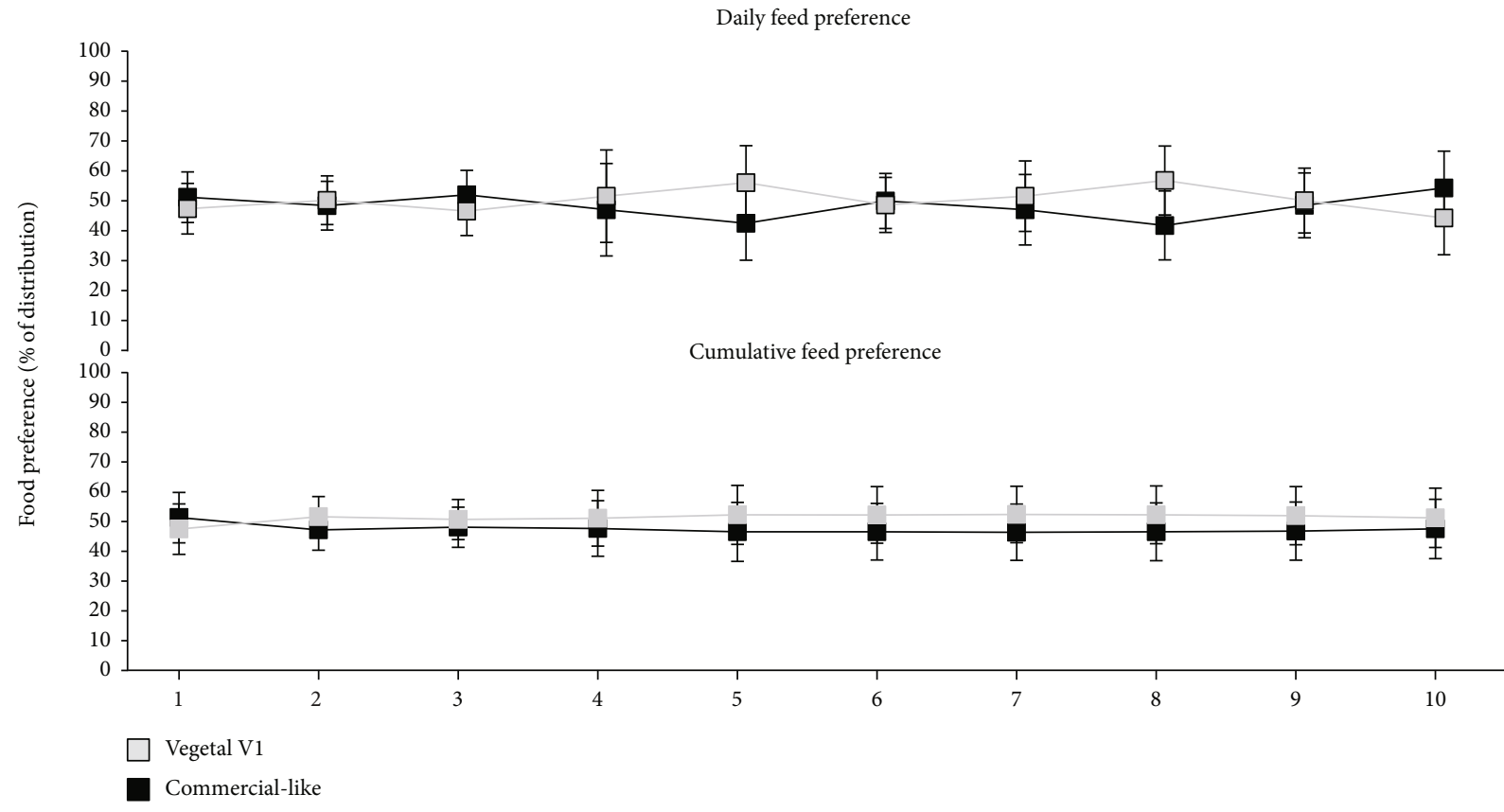

(c)

Figure 1: Preference test during 10 days of rainbow trout (RT) fed for 30 days with commercial (c), V1 or V2 diets. (a) Representative daily (upper) and cumulative (lower) feed preference for V1 and V2 diets for RT fed for 30 days with the C diet. (b) Representative daily (upper) and cumulative (lower) feed preference for V2 and commercial diet for RT fed for 30 days with the V1 diet. (C) Representative daily (upper) and cumulative (lower) feed preference for V1 and C diets for RT fed for 30 days with the V2 diet. An asterisk indicates a significant difference between the two diets as determined by a $t$-test $(P<0.05)$. Results are expressed in $\%$ of distribution as mean \pm S.E. $(n=5$ tanks).

Bretonneux, France) following the TRIzol manufacturer's instructions. Total RNA ( $2 \mu \mathrm{g})$ was used for cDNA synthesis. RNA purity was tested by the optical density (OD) absorption ratio (OD $260 \mathrm{~nm} / 280 \mathrm{~nm}$ ) using a NanoDrop 2000c (Thermo, Vantaa, Finland), and only samples with an OD $260 \mathrm{~nm} / 280 \mathrm{~nm}$ ratio $>1.8$ were used for analysis. The Super-Script III RNAse H-Reverse transcriptase kit (Invitrogen) was used with random primers (Promega, Charbonniéres-les-bains, France) to synthesize cDNA in a final volume reaction of $20 \mu \mathrm{l}$, according to the manufacturer's instructions. QPCR assays were performed with the Roche Lightcycler 480 system (Roche Diagnostics, Neuilly-surSeine, France). The reaction mix was $6 \mu \mathrm{l}$ per sample, including $2 \mu \mathrm{l}$ of diluted cDNA template $(1: 10), 0.12 \mu \mathrm{l}$ of each primer $\left(10 \mu \mathrm{moll}^{-1}\right), 3 \mu \mathrm{l}$ of Light Cycler $480 \mathrm{SYBR}^{\circledR}$ Green I Master mix and $0.76 \mu \mathrm{l}$ of DNAse/RNAse-free water (5 Prime GmbH, Hamburg, Germany). The QPCR protocol was initiated at $95^{\circ} \mathrm{C}$ for $10 \mathrm{~min}$ for the initial denaturation of the cDNA and hot-start Taq-polymerase activation, followed by 45 cycles of a two-step amplification program $\left(15 \mathrm{~s}\right.$ at $95^{\circ} \mathrm{C} ; 10 \mathrm{~s}$ at $\left.60^{\circ} \mathrm{C}\right)$. Melting curves were monitored systematically (temperature gradient $0.11^{\circ} \mathrm{C}$ per $\mathrm{s}$ from 65 to $97^{\circ} \mathrm{C}$ ) at the end of the last amplification cycle to confirm the specificity of the amplification reaction. Duplicate wells were used for each sample and negative controls were included in all reactions, consisting in wells containing RNA samples and water instead of cDNA. Efficiency of all QPCR reactions was $93-100 \%$ and $R^{2}$ was $0.96-1$. Data were extrapolated from standard curves and normalized to the housekeeping genes after validation; compared to $18 \mathrm{~s}$ and actin, elongation Factor $1 \alpha$ gene $(e e f 1 \alpha)$ was the most relevant gene which was stable between tissues. Also, due to this high stability and higher copy of the gene eef $1 \alpha$ is quite suitable reference gene for the normalization analysis in RT and relevant for nutritional experiment or hypothalamus transcriptional analysis [11, 27]. However, in tongue, Keratin $8 \mathrm{~b}(k r t 8 b)$ was additionally evaluated as a reference gene that it is specifically expressed in the epithelia of taste buds in mammals, by also in fish [43]. The aim was to ensure that differences in expression are not caused by a different number of taste buds being coincidentally included in each sample. The expression of $k r t 8 b$ in this experiment was compared with that of $18 \mathrm{~s}$ and eef $1 \alpha$, and a similar pattern of raw data (that is, non-normalized) was observed. Therefore, both $k r t 8 b, 18 s$ and eef $1 \alpha$ were chosen for normalization in tongue tissue (Figure 3). Relative expression of the target genes was determined by the $\Delta \Delta \mathrm{CT}$ (Cycle Threshold) method [44]. Mean \pm S.E.M. values for each group are expressed in fold changes relative to organ (tongue for Figure 4) or C diet (Figures 3 and 5 and supplemental information 2). The mRNA sequences of RT used in this study are available in the NCBI and Ensembl genome browser. By comparing other species (teleosts, rodents and humans), we found the available sequences of genes in RT with variant paralogs and accession numbers (Table 4). Mammalian species were chosen because their fatty acid receptors in particular have been characterized and studied. The zebrafish (danio rerio) model (the main fish specie model in the world, 


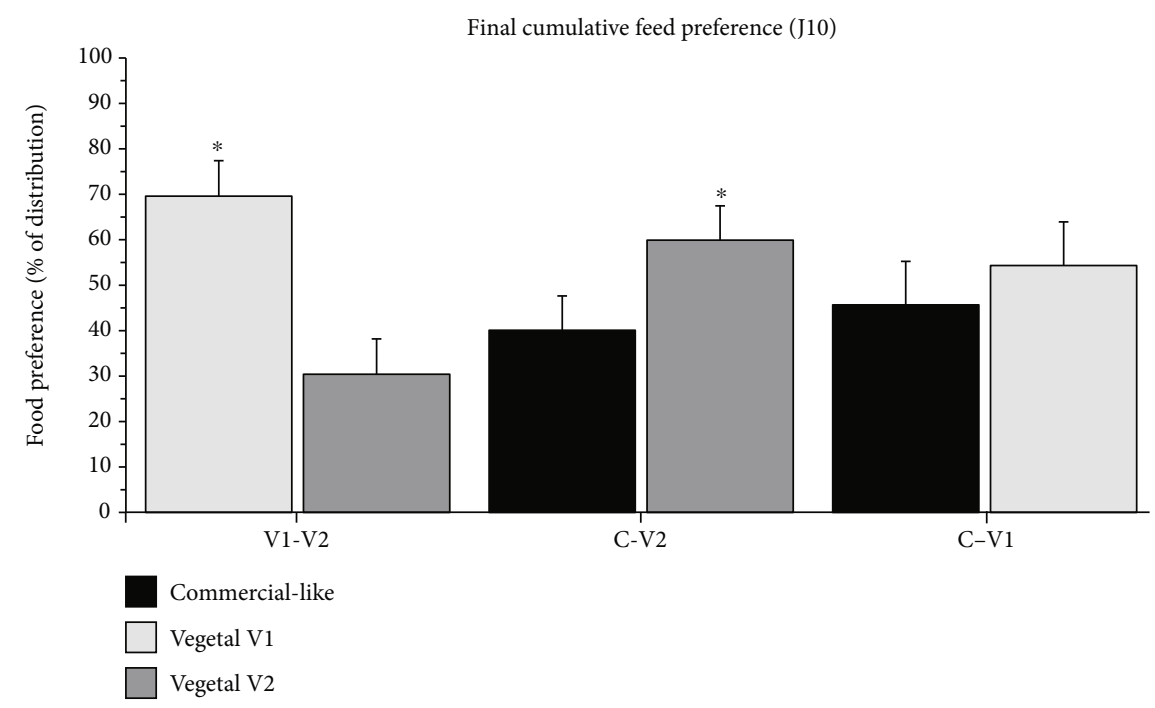

(a)

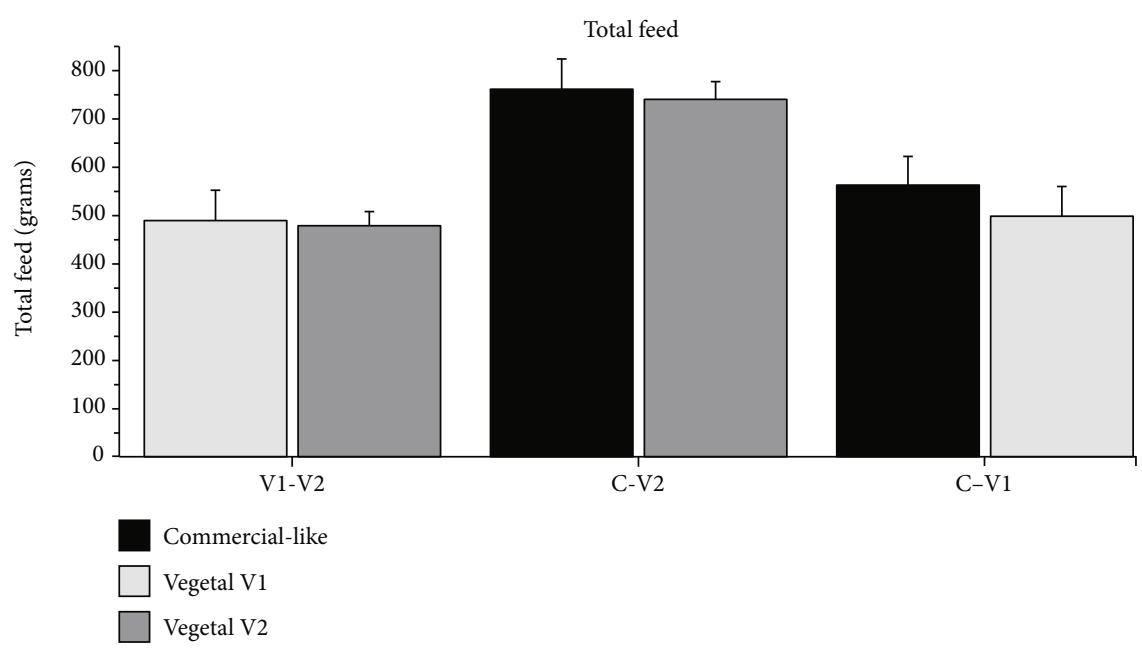

(b)

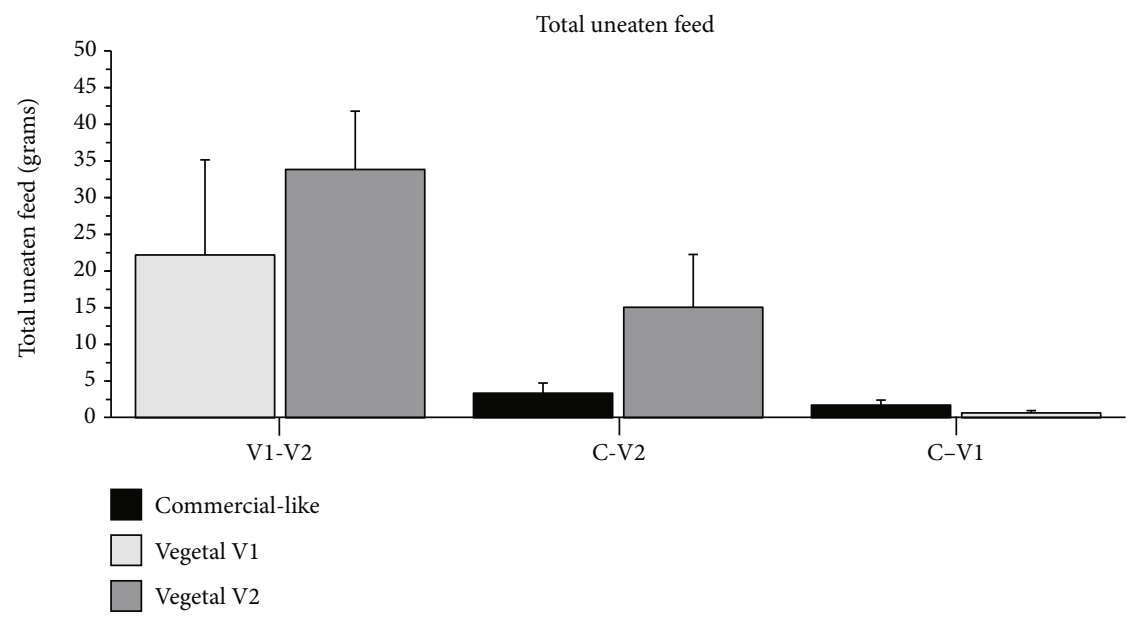

(c)

FIGURE 2: Total feed preference, feed consumed and uneaten feed pellets at the end of the preference trial. (a) Representative final cumulative feed preference at the end of the preference trial (10 days). (b) Representative total feed consumed at the end of the preference trial in all diets. (c) Representative total uneaten pellets at the end of the preference trial in all diets. An asterisk indicates a significant difference between the two diets as determined by a $t$-test $(P<0.05)$. Results are expressed in \% (of total feed distribution) for preference $(\mathrm{a})$ and $\mathrm{g}$ for total (b) and uneaten feed (c) as mean \pm S.E. ( $n=5$ tanks). 


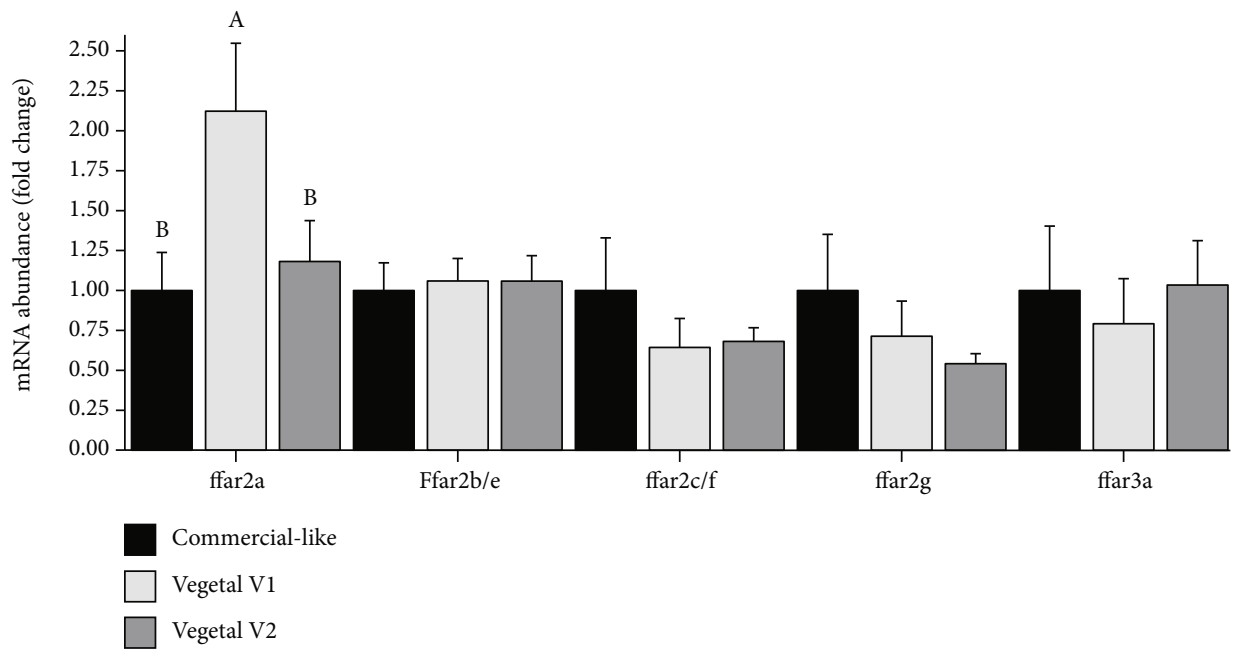

(a)

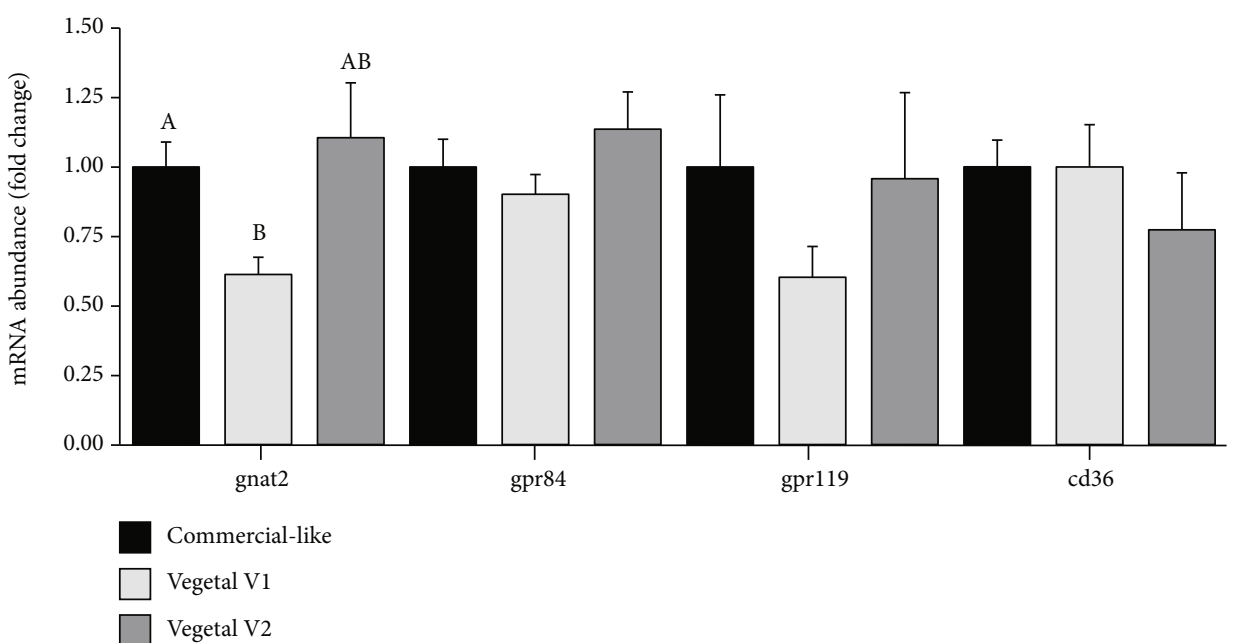

(b)

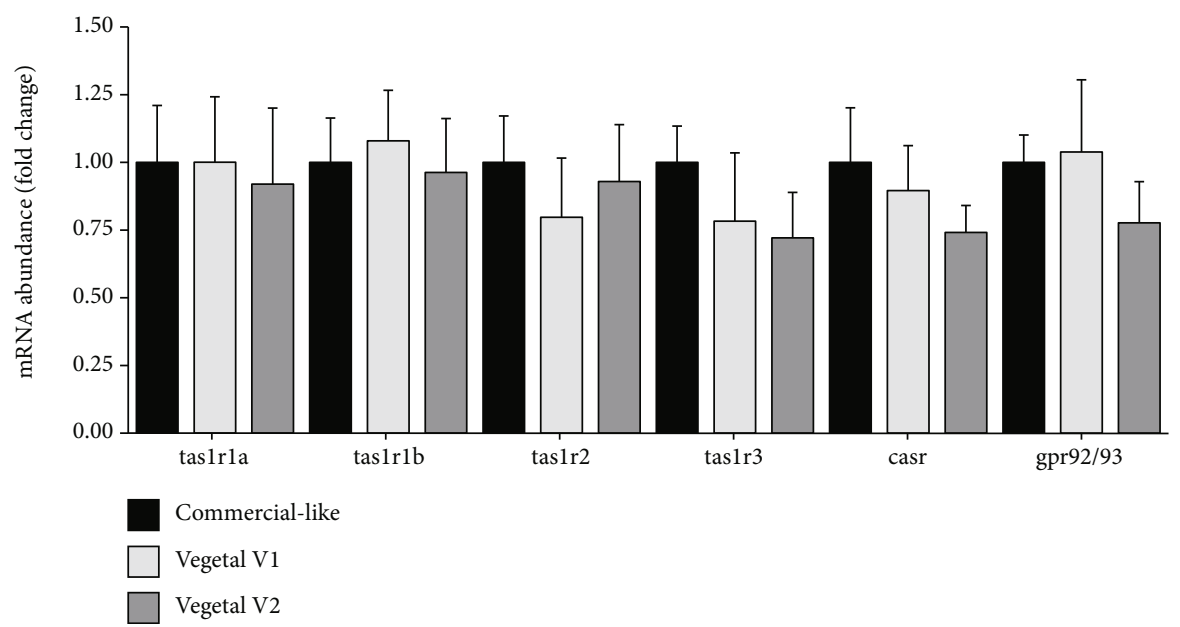

(c)

FIGURE 3: Gene expression profiling related to taste receptors in the tongues of rainbow trout (RT) fed for 30 days with commercial (C), V1 and V2 diets. Relative gene expression measured by RT-PCR of ffar2a, ffar2b/e, ffar2clf, ffar2g, ffar3a, ffar3b, gnat2, gpr84, gpr119, cd36, tas1r1a, tas1r1b, tas1r2 tas1r3, casr and gpr92/93 in the tongue of RT fed for 30 days with $\mathrm{Cl}, \mathrm{V} 1$ and $\mathrm{V} 2$ diets. Values are expressed as group mean $\pm \mathrm{SEM}$; fold change $v$. C diet for all genes. One-way ANOVA, Tukey post hoc; Different lower case letters indicate significant differences $(P<0.05)$ among diets $(n=6)$. 


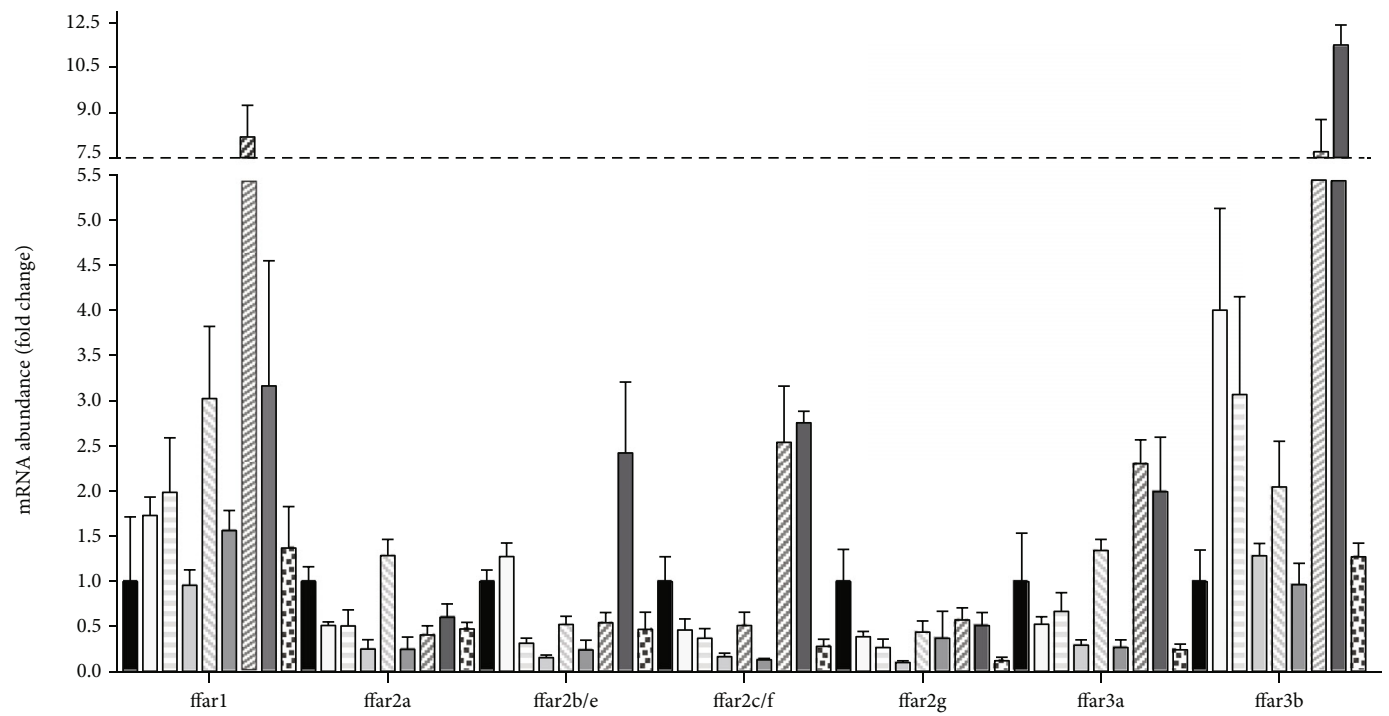

(a)

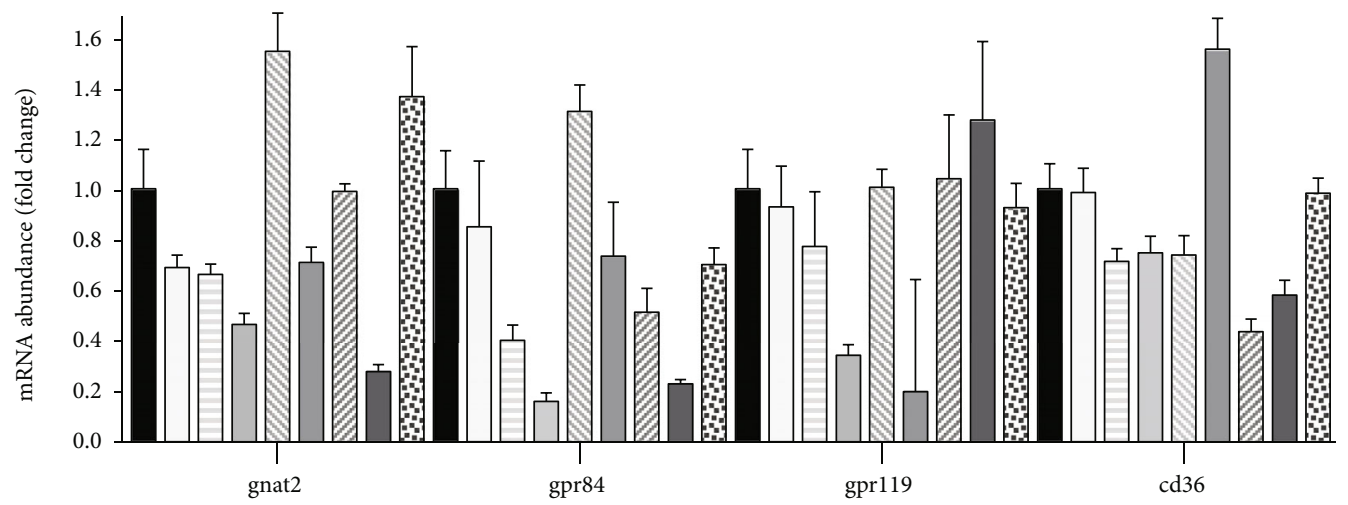

(b)
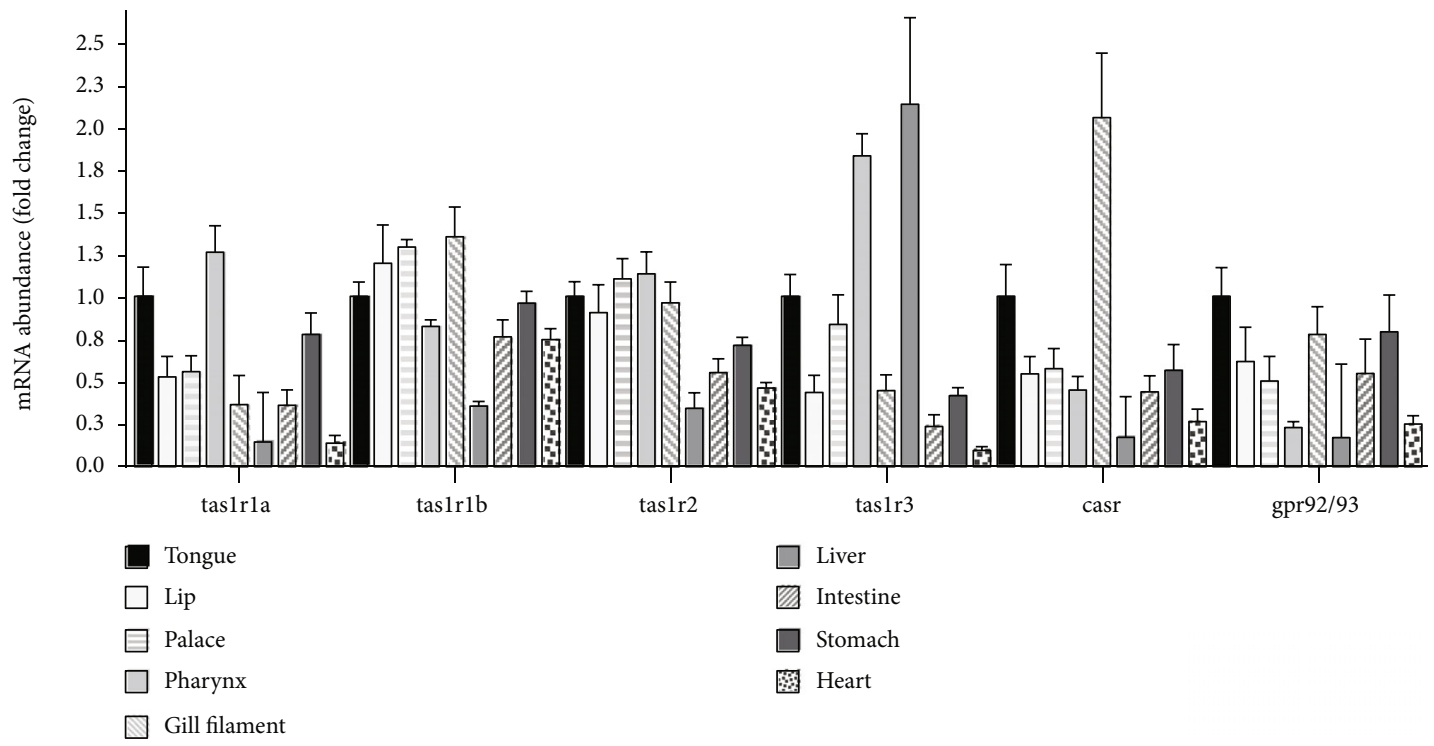

(c)

FIGURE 4: Gene expression related to taste receptors in different organs of rainbow trout (RT) fed for 30 days with a commercial diet (C). Relative gene expression measured by RT-PCR of ffar1, ffar2a, ffar2ble, ffar2clf, ffar2g, ffar3a, ffar3b, gnat2, gpr84, gpr119, cd36, tas1r1a, tas1r1b, tas1r2 tas1r3, casr and gpr92/93 in the tongue, lip, palate, pharynx, gill filament, liver, intestine, stomach and heart of RT fed for 30 days with the $\mathrm{C}$ diet. Values are expressed as group mean \pm SEM; fold change $v$. tongue for all genes $(n=6)$. One-way ANOVA, Tukey post hoc. 


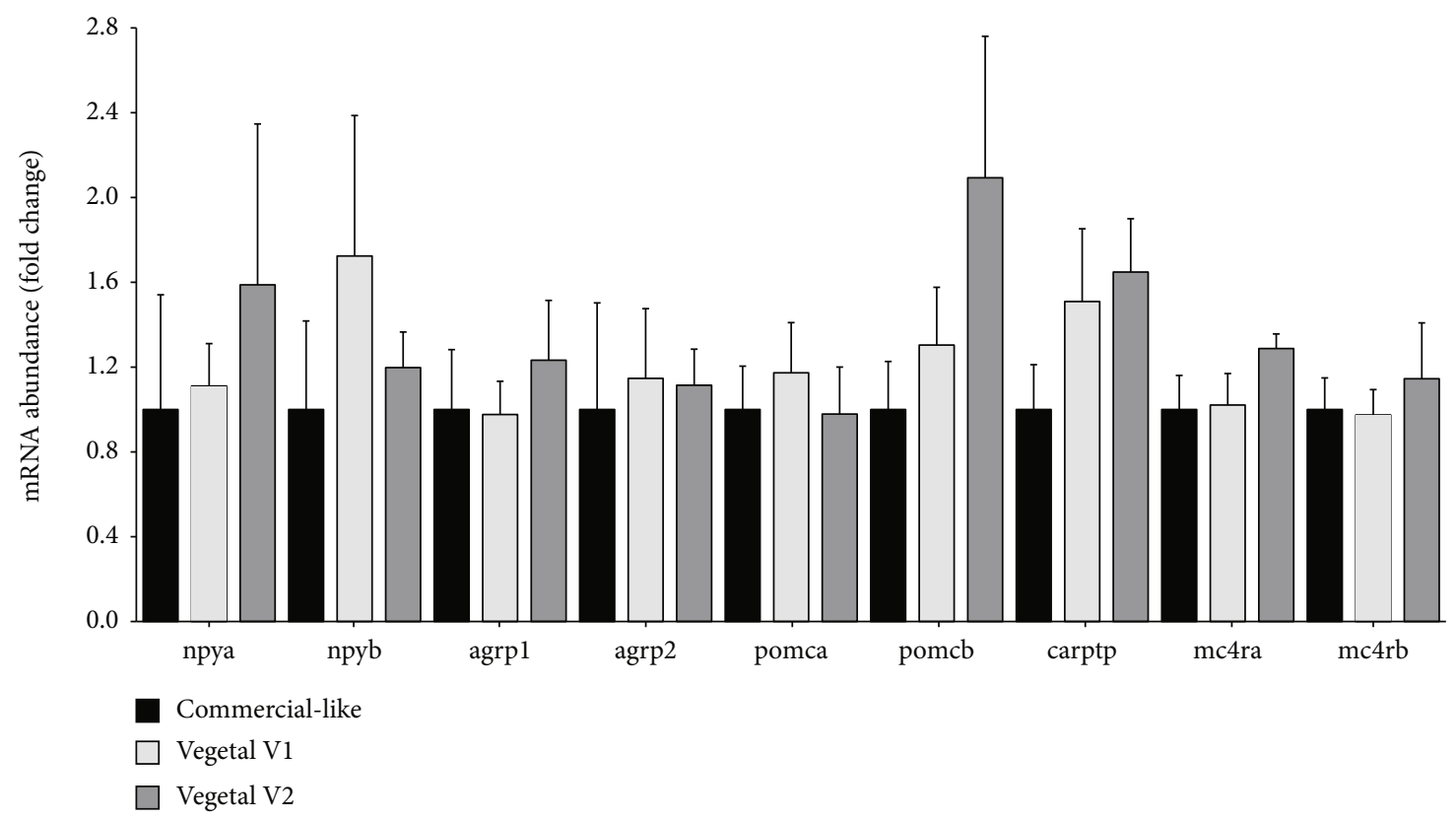

FIGURE 5: Gene expression related to FI mechanisms in the hypothalamus of rainbow trout (RT) fed for 30 days with commercial (C), V1 and V2 diets. Relative gene expression measured by RT-PCR of npya, npyb, agpr1, agrp2, pomca, pomcb, cartpt, mc4ra and $m c 4 r b$ in the hypothalamus of RT fed for 30 days with $\mathrm{C}, \mathrm{V} 1$ and V2 diets. Values are expressed as group mean \pm SEM; fold change $v$. commercial diet for all genes; one-way ANOVA, Tukey post hoc; $(n=6)$.

where the genome and receptors have already been studied and quantified) allowed us to compare the RT receptors with another fish species (and to quantify the differences between species (between fish species or fishes $v$. mammals). All gene sequences of RT used were identified by in silico analysis from the Genomicus software program, version 100.01 (http://www.genomicus.biologie.ens.fr) and Ensembl (http://www.ensembl.org, Ensembl Release 102; November 2020, RT genome available). They were queried against the human, rodent and zebrafish genomes using the BLAST tool in Ensembl and in NCBI (https://blast.ncbi.nlm.nih.gov/ Blast.cgi) to confirme gene identification (Table 5(a)). For paralog genes (such as ffar2), the percentage of similar sequences was determined by alignment of the mRNA of RT genes using MUSCLE software (http://www.ebi.ac.uk/ Tools/msa/muscle) and compared to other species (Table 5(b)). In the case of genes that possesed numerous paralogs, when some of them presented high similar sequences (> 95\%), we designed primer pairs to amplify paralogs together in order to not distinguish the expression of each of them (ffar2 for paralog $b$ and $e, c$ and $f$ ).

2.8. Statistical Analysis. Statistical analyses were performed using R software (v3.5.2)/R Commander package. Data were tested for normality of distribution and homogeneity of variance using the Shapiro-Wilk test and Bartlett's test, respectively. If these two assumptions were met, comparisons among groups were analyzed using one-way ANOVA followed by Tukey's HSD as a post hoc test. If the data were not normally distributed, Levene's test for homogeneity of variance was used. When variables did not follow either of the assumptions of normal distribution or equal variance, a non-parametric Kruskal-Wallis test was used, followed by a pairwise Wilcoxson test was used. To evaluate the effect of the diet on time course changes in daily and cumulative feed preferences for each diet (Figure 1), values were analyzed using frequency analysis (one-sample $t$-tests). The total food consumed and total uneaten pellets, values were analysed by $t$-tests. All data are expressed as mean \pm S.E. treatment (diet) effects and interactions were considered statistically significant at $P<0.05$.

\section{Results}

3.1. Growth Performance and Feed Parameters during the Feeding Trial. Fish growth performance and feed parameters at the end of the feeding trial are presented in Table 3. For all tanks and diets (with no significant difference for initial biomass and number of fish per tank), fish growth increased by $>50 \%$ after 4 weeks feeding, and no mortality was recorded. $\mathrm{C}$ and V1 diets showed higher BWG than the V2 diet $(P<0.05 ; \mathrm{C} v$. V2). This represented a DGC of 2.54 $\pm 0.15 \%$ and $2.51 \pm 0.20 \%$ for $\mathrm{C}$ and V1 diets of their initial body weight compared to $1.85 \pm 0.38 \%$ for the V2 diet $(P<0.05 ; \mathrm{C} v$. V2). Daily FI was higher for the V1 diet (but the same as the control diet) compared to the V2 diet $(P<0.05)$. Feed efficiency was $>1.0$ for the $\mathrm{C}$ diet $(1.05$ $\pm 0.02)$ and significantly lower for the V2 diet $(0.89 \pm 0.08)$ but not for the V1 diet $(0.90 \pm 0.06)$. The DEI was higher for the V2 diet compared to the $\mathrm{C}$ diet $(P<0.05)$. Total FI was higher for the V1 diet compared to the V2 diet $(P<0.05)$ and the uneaten feed was very low for the three groups $(<1.5 \%$ of total distribution). No diet interaction was observed for growth and feed parameters. 


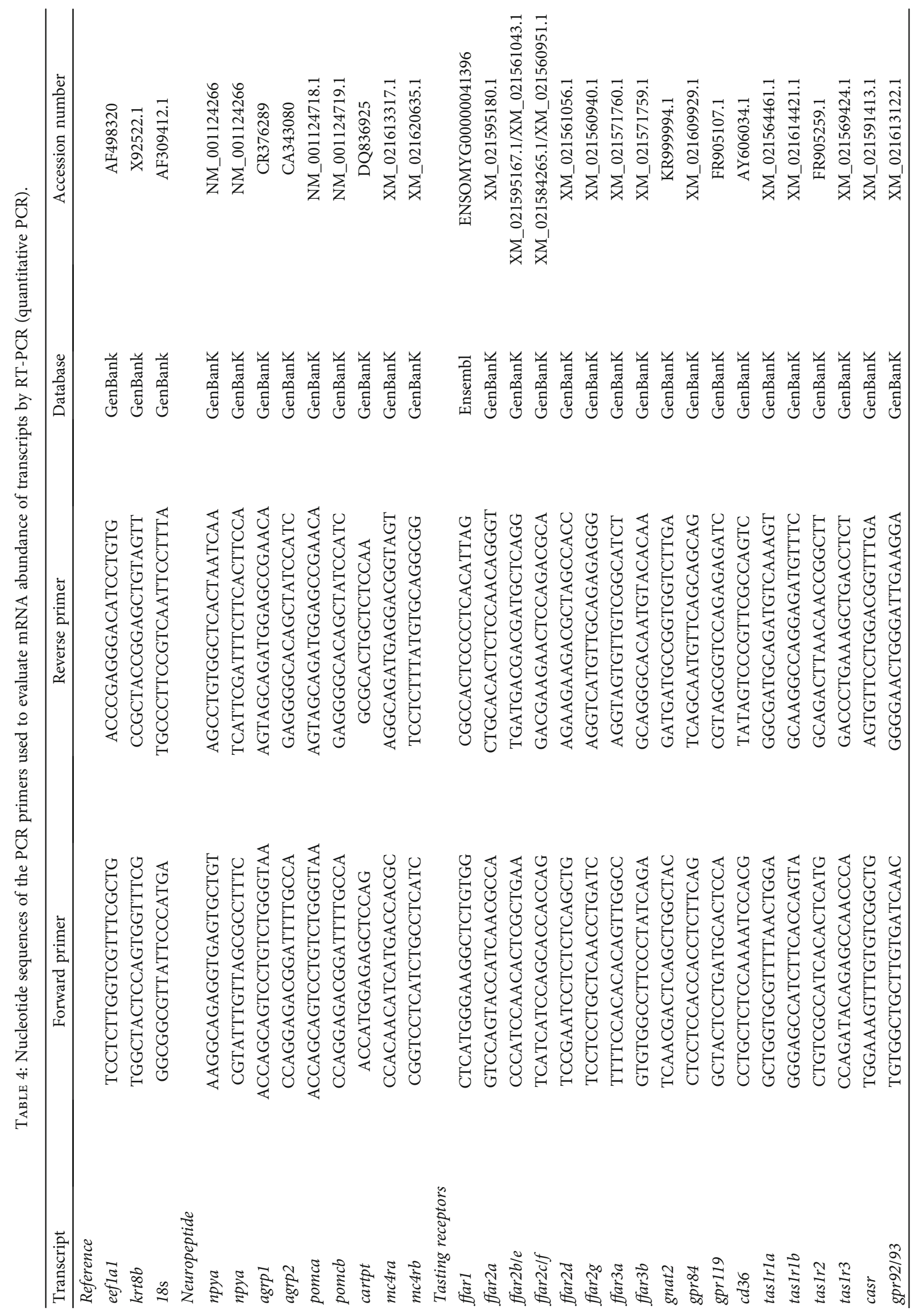


TABLE 5: (A) In silico analysis of FFAR1, 2, 3 and 4 identified in rainbow trout (RT) and compared to human, mouse and zebrafish species and identification of sequence homology of FFAR2 (using MUSCLE software) RT paralogs compared to human, mouse and zebrafish FFAR1, FFAR4 (B) or FFAR2 sequence (C).

(a)

Human (Homo sapiens)
GPR40 (FFAR1)
GPR43 (FFAR2)
GPR120 (FFAR4) (FFAR3)

(b)

\begin{tabular}{lccc}
\hline & Human (Homo sapiens) & Mouse (Mus musculus) & Zebrafish $($ danio Reno) \\
\hline Human FFAR2 & $\mathbf{1 0 0 . 0 0}$ & 84.55 & 43.84 \\
Mouse FFAR2 & 84.55 & $\mathbf{1 0 0 . 0 0}$ & 45.99 \\
Zebrafish FFAR2 & 43.83 & 45.99 & $\mathbf{1 0 0 . 0 0}$ \\
Rainbow trout FFAR2a & 47.15 & 46.20 & 59.49 \\
Rainbow trout FFAR2b/e & 45.43 & 46.34 & 57.54 \\
Rainbow trout FFAR2clf & 42.21 & 41.88 & 47.23 \\
Rainbow trout FFAR2d & 44.88 & 44.88 & 47.35 \\
Rainbow trout FFAR2g & 45.65 & 46.27 & 62.73 \\
\hline
\end{tabular}

(c)

\begin{tabular}{lccccc}
\hline & Human (Homo sapiens) & Mouse (Mus musculus) & & Human (Homo sapiens) & Mouse (Mus musculus) \\
\hline Human FFAR1 & $\mathbf{1 0 0 . 0 0}$ & 83.00 & Human FFAR4 & $\mathbf{1 0 0 . 0 0}$ & 86.43 \\
Mouse FFAR1 & 83.00 & $\mathbf{1 0 0 . 0 0}$ & Mouse FFAR4 & 84.55 & $\mathbf{1 0 0 . 0 0}$ \\
Rainbow trout FFAR2a & 29.75 & 28.67 & Rainbow trout FFAR2a & 19.38 & 20.49 \\
Rainbow trout FFAR2b/e & 30.60 & 28.93 & Rainbow trout FFAR2b/e & 21.23 & 23.04 \\
Rainbow trout FFAR2clf & 31.01 & 30.48 & Rainbow trout FFAR2clf & 20.75 & 22.6 \\
Rainbow trout FFAR2d & 28.39 & 28.77 & Rainbow trout FFAR2d & 20.68 & 21.49 \\
Rainbow trout FFAR2g & 28.57 & 27.18 & Rainbow trout FFAR2g & 18.09 & 20.81 \\
\hline
\end{tabular}

3.2. Feed Preference and Feed Parameters during the Preference Trial. The daily and cumulative feed preferences for three dietary groups are presented in Figures 1 and 2 for final cumulative feed preference and feed parameters. For RT fed for 30 days with the $\mathrm{C}$ diet (Figure 1(a)), feeding preference between V1 and V2 dietary groups revealed that fish had an early and constant preference for the V1 diet with $61.97 \%$ of daily feed preference, and $70.87 \%$ at the end of the preference trial (day 10) compared to the V2 diet. The final cumulative feed preference for the V1 diet compared to the V2 diet was $69.6 \%(P<0.05)$.

Fish fed for 30 days with the V1 diet during the feeding trial had an early daily feed preference for the V2 diet compared to the $\mathrm{C}$ diet (Figure 1(b)). The final cumulative feed preference after 10 days was $59.92 \%$ for the V2 diet compared to $40.08 \%$ for the $\mathrm{C}$ diet (Figure $2(\mathrm{a})$ ). Hovewer, daily feed preference revealed that fish had changed their feed preference (for the $\mathrm{C}$ diet), from day 8 until the end of the preference period $(61.23 \% ; P<0.05)$.

For fish fed for 30 days with the V2 diet, daily and cumulative feed preferences showed that fish had no feed preference between the two diets $\mathrm{C}$ and V1 (Figure 1(c)). The final cumulative feed preference revealed no feed preference for fish between the $\mathrm{C}$ and V1 diets (Figure 2(a)) with $48.16 \%$ v. $51.84 \%$ for $\mathrm{C}$ and $\mathrm{V} 1$ diets, respectively.

The total consumed and uneaten feeds are presented in Figures 2(b) and 2(c) at the end of the preference period (10 days). Even if daily or cumulative feed preference revealed preference for $\mathrm{V} 1$ and $\mathrm{V} 2$ diets for fish fed for 30 days with the $\mathrm{C}$ and V1 diets, respectively, total feed consumed and uneaten feed (all tank for each diet) were not different for all three diets choices. 
3.3. Hypothalamus Neuropeptide Gene Expression Profiling. Different gene expression values in the hypothalamus are presented in Figure 5 as fold changes of relative gene expression over the control group (C diet). The gene expression of orexigen $n p y$ ( $a$ and $b$ paralogs) and agrpl and 2 peptide was not different among the three diets. No differences were also observed among the three diets for mRNA expression of anorexigenic pomc ( $a$ and $b$ paralogs) and cartpt peptide. The gene expression of MC4R ( $a$ and $b$ paralogs) gene encoding receptor up-regulated by anorexigenic POMC peptide and down-regulated by AGRP orexigenic peptide was not different in the hypothalamus of fish fed during the feeding trial with the three diets $6 \mathrm{~h}$ after last meal.

\subsection{Identification of the FFAR Sequence in the Rainbow} Trout Genome. The identification and homology of the FFAR sequence of selected species (human, mouse and zebrafish) compared to RT using the newly available RT annotated genome assembly, Omyk_1.0. are presented in Table 5.

Our in silico analysis revealed that the ffar4 (encoding GPR120) gene sequences was not reported in the RT genome (Table 5(a)). For ffar 1, ffar2 and ffar3, we found 1 sequence, 8 and 2 paralogs, respectively. High similar sequences homology (> 95\%) were observed for ffar2 para$\operatorname{logs}$ (paralog $b$ and e, $\mathrm{c}$ and $\mathrm{f}$ and $\mathrm{g}$ ). All ffar2 paralogs of RT displayed $<31 \%$ of homology with ffar 1 and $<23 \%$ of homology with ffar4 sequence of humans and rodents (Table 5(b)). By comparing the sequence homology of ffar2 paralogs of RT, we identified the percentage of homology with the human, mouse and zebrafish ffar2 sequence (Table 5(c)). All ffar2 paralog genes of RT displayed low sequence homology with humans $(<48 \%)$, rodents $(<47 \%)$ but also for zebrafish $(<63 \%)$.

3.5. Taste Receptor Gene Expression Profiling in RT. The gene expression of taste receptors in several tissues of RT are presented in Figure 4. All mRNA sequence encoding taste receptors were detected (except for ffar $2 d$ ), at different levels, in all of the tissues studied (tongue, lip, palate, pharynx, gill filament, liver, proximal intestine, stomach and heart).

The gene expression of the FFAR family is presented in Figure 4(a). The mRNA level of ffarl was very low expressed in all organs (CT means $>31$ ) with a higher mRNA level for the intestine with 8 folds compared to the tongue tissue with the lowest expression and very low detection (CT means $>34$ ). The gene expression of all paralogs of ffar 2 transcripts was predominantly expressed in the tongue $(P<0.05)$. The gene expression of all paralogs of ffar 2 transcripts were lowest in liver tissue and as expected in the heart tissue (used as a negative control). Individually, gene expression of ffar2a paralog was highest in the gill filament, stomach and proximal intestine for ffar $2 b / e$ and ffar $2 c / f$, respectively, and in the tongue for ffar $2 g$. The gene expression of ffar 3 paralogs was expressed in numerous organs with a higher mRNA level for the two paralogs in the intestine and stomach with 14 folds compared to the tongue tissue with a very low expression. The detection of the mRNA level of the ffar $3 b$ gene was very low compared to the stomach and intestine tissues. The gene expression of gnat2, gpr84, gpr119 and $c d 36$ are shown in
Figure 4(b). Compared to the FFAR family, gene expression of these four taste receptors was not predominantly expressed in the tongue and not in the stomach (except for gpr119) compared to ffar2 (b/e and $c / f$ ) and ffar3 ( $a$ and $b)$. Gene expression of gnat2 was the highest in the gill filament followed by the heart. The highest gpr 84 mRNA abundance was observed in the gill filament, followed by the tongue, and the lowest mRNA level was detected in the pharynx. Gene expression of gpr84 and $c d 36$ was homogenous between tissues with the highest level in the liver and stomach for gpr84 and $c d 36$, respectively. Gene expression of amino acid taste receptors are shown in Figure 4(c). As in the FFAR family, gene expression of amino acid taste receptors was mainly expressed in the tongue $(P<0.05)$. Heart tissue used as a negative control expressed very low mRNA levels of amino acid taste receptors (excepted tas $1 r 1 b$ ). Individually, gene expression of tas $1 r 1 a$ and $b$ was highest in the pharynx and gill filament for paralogs a and $b$, respectively, and the mRNA level was lowest in the liver. This was also the case for tas1r2, casr and gpr92/93 whereas the liver was the tissue which had the highest expression of tas1r3. Thus for taslr1a, the gene expression of tas $1 r 2$ and tas $1 r 3$ was predominantly expressed in the pharynx (and liver for tas1r3) and for tas $1 r 1 b$, gene expression of the casr gene was predominantly expressed in the gill filament.

3.6. Influence of the C, V1 and V2 Diets on the Taste Receptor Gene Expression Profiling in the Tongue of RT. The gene expression of taste receptors in the tongue of RT are presented in Figure 3 (except for ffarl transcripts, which was too weakly expressed in tongue tissu with a strong heterogeneity between replicats preventing data exploitation). Whereas gene expression of ffar3a (ffar $3 b$ was poorly detected to conclude any difference) was not affected by diet, gene expression of ffar2 was regulated by V1 diet with upregulation for ffar2a compared to the C diet (Figure 3(a); $P<0.05)$. Gene expression of gnat2 was downregulated by the $\mathrm{V} 1$ diet compared to the $\mathrm{C}$ diet $(P<0.05)$. For amino acid receptors, no change was observed between diets (Figure 3(c)).

\section{Discussion}

In mammals, taste provides critical information about the quality and nature of nutrients, leading to specific eating responses (consumption or avoidance), fundamental for their growth and survival [17]. In fishes, the genetic basis underlying the formation of food habits is largely unknown [45]. In the present study, our results highlighted the fact that juvenile RT have the fundamental mechanisms for oro-gustatory perception of nutrients related to different diet composition. Surprinsigly, our findings revealed that the V2 diet affected the growth performance of RT. In contrast to our previous study [10] which showed feed preference for a diet enriched in $\omega$-3 LC-PUFA (DHA and EPA), this study revealed an alteration of feeding behavior for fish fed with the V2 diet for 30 days. During the feeding trial, FI and feed efficiency were affected but without affecting the mRNA level of neuropeptide transcripts suggesting either a 
palatability or metabolic disorder (alteration in nutrient metabolism) or both. During the preference trial the V1 diet was consumed only a little compared to the V2 diet for fish fed with the $\mathrm{C}$ diet during the feeding trial. The amino acid profile was the same between V1 and V2 diets ruling out an amino acid preference effect. For fish fed with the V1 diet during the feeding trial, we observed a change in preference after 8 days for the $\mathrm{C}$ diet compared to the V2 diet. These results suggested a concern both with the palatability and with metabolism. No preference was observed between $C$ and $\mathrm{V} 1$ diets for fish fed with the V2 diet during the feeding trial. This suggests that the DHA proportion in the diet (same as in C and V2 diets) or absence of FM/FO in the V2 diet (also in the V1 diet) was not the main causes of the feeding behavior alteration for fish fed with the V2 diet. Also, in our previous preference [10] and feeding trials [12], the level of $\omega-3$ LC-PUFA did not affect growth or preference of the fish. It is important to mention that the $\omega-3$ LC-PUFA was provided by marine animal oil (fish oil). Moreover, algae oil was the only raw material absent in $\mathrm{C}$ and V1 diets. The algae oil added in the V2 diet would be the main cause on the alteration of FI, feed efficiency and preference of RT during the two trials. Additional studies will be required to determine if the algal strain, $\omega-3$ LCPUFA level or source (animal $v$. vegetal oil) are involved in this lack of palatability.

The lack of palatability for the V2 diet directly influences food preference, which makes it difficult to interpret results for the preference trial. However, in view of preferences that have early emerged during the preference trial, it remains possible to make some assumptions. Fish fed for 30 days with the $\mathrm{C}$ diet had an early and constant preference for V1 $v$. V2 diets with high consumption for both diets. In contrast to a previous study [42], this result could suggest that presence of ALA or linseed oil in the diet composition did not influence preference for a diet. This conclusion is confirmed by the observation that no preference was observed between $C$ and $\mathrm{V} 1$ diets for fish fed with the V2 diet for 30 days. In addition, these two dietary groups have demonstrated that the influence of a past diet did not influence the choice of an alternative diet. Hovewer, the V1 diet led to a preference during 8 days for the V2 diet compared to the $C$ diet. This may have been because the lack of palatability of the V2 diet kept the fish away from that feeder. The preference observed for this group could suggest the influence of a past diet for feed preference. RT which had consumed a plant-based diet would be attracted to a diet with very similar composition (V1 $v$. V2 diet), despite a diet with FM/FO known to be appreciated by the fish. The lack of palatability of the V2 diet supported this interpretation. Conversely, RT which had consumed the diet with FM/FO would not immediately be attracted to a diet with the same FA profile (V2 diet). These results could suggest the importance of raw materials more than nutrients for the adaptation of RT to a new diet.

At the molecular level, the oro-sensory detection for nutrients by taste receptors could also explain these results. Although a difference in growth performance was observed for the V2 diet in the feeding trial compared to V1 and C diets, RT had vigorously consumed this diet for 30 days, allowing us to make assumptions in the regulation of taste receptors of fish fed with these three diets. Among numerous functions [13], taste receptors help to coordinate the response to the meal, and contribute to the regulation of satiation [16]. Numerous membrane proteins are reported to be expressed in taste receptor cells named tast bud cells. These proteins exhibit a predicted seven transmembrane topology and sequence motifs characteristic of $G$ proteincoupled receptors including FFAR, e.g. FFAR1, 2, 3 and 4 [20] or CD36 [22] for FA sensing and T1R for amino acid and sugar sensing. However, research on the detection of nutrients has mainly been conducted on rodent models as well as on human taste bud cells [23] but remains poorly studied in fishes [24]. Specifically, as for other senses of taste, the initial stage of FA sensing in the taste bud cells arises by the effective expression and distribution of fat taste receptors allowing the binding and detection of fatty acids [46].

Our findings surprisingly revealed that sequence encoding GPR120 (ffar4) genes was absent in the RT genome (Ensembl) and very low expressed for ffarl in tongue tissue (CT means $>34$ ). This finding was surprising because the two receptors FFAR1 and FFAR4 are reported to be involved in oro-gustatory perception for fat tasting transduction in mammals [21] and are also implicated in various biological and physiological functions such as energy regulation, metabolism, immunological homeostasis, and neuronal functions [29]. Moreover, this present study revealed numerous ffar2 and ffar3 paralog genes of RT, highly expressed in the taste organ and modulated by diet suggesting an important role of these other FFAR in the orogustatory perception for fat tasting in fishes. First, in silico analysis revealed that all ffar 2 paralog genes of RT displayed low sequence homology along with humans, rodents and also zebrafish for ffar2, 3 and 4 sequence genes. These data suggested that the role of FFAR1 and FFAR4 in mammals, especially those involved in oro-gustatory perception for fat taste transduction, could be by other receptors in fishes. In particular, we observed that high FFAR2 duplications in RT (seven copies) were mainly expressed in the tongue and two duplications of FFAR3 were mainly expressed in digestive tissues. Knowledge on these taste receptors in fishes are scarce, and the role of the localities of FFAR2 and FFAR3 have to be compared with mammals.

In mammals, the FFAR2 receptor is expressed in adipose tissue, intestines, islet cells of the pancreas, and immune tissues, and is involved in the regulation of appetite and insulin signaling. It contributes to the maintenance of energy homeostasis and plays a role in the immune response via FA-induced signaling [47]. In RT, ffar2_ble and ffar2_clf could fulfil these physiological functions involving intestine and stomach tissues. In contrast, whereas FFAR2 was not reported in mammals to take part in oro-sensory detection in the oral cavity [29], ffar2a seems implicated in the detection and regulation of fat tasting in fishes as revealed by diet and upregulation by a vegetal diet. Moreover, ffar2a transript was no regulated by V2 diet which suggested a role in oro-gustatory fat perception especially for ALA (mainly fatty acid in V1 diet, less than $1.2 \%$ in $\mathrm{C}$ and $\mathrm{V} 2$ diet). 
Gene expression of FFAR3 was quantified in various tissues including the intestine, gut, pancreas, thymus, adipose tissues, immune cells and peripheral nervous systems of mammals. This receptor has been reported to contribute in the regulation of energy homeostasis via FA-induced signaling [48]. In RT, the gene expression of ffar 3 could reflect the same physiological functions as reported in mammals. The very low gene expression of ffar3a and no expression of ffar $3 b$ in the oral cavity and the absence of regulation by diet in our study in the tongue also suggested the non-involvement of FFAR3 in fat sensing. To conclude for the FFAR family, our results could suggest that some paralogs of FFAR2 could be involved in the FA sensing system. We also make the assumption that the role of FFAR1 and FFAR4 in mammals, especially those involved in oro-gustatory perception for FA sensing transduction, [21], could be undertaken by some of the FFAR2 paralogs.

In mammals, FFAR are known to have high affinity for $\omega$ 3 LC-PUFA [49]. This affinity was mainly shown by FA level differences between the three diets in our study. It is known that $\omega$-3 LC-PUFA are most sensitive to oxidation [50]. We have recently revealed the presence of some selected enzymatic and non-enzymatic oxidation metabolites of $\omega$-3 LCPUFA in the brain of RT and the modulation of the brain lipid content by distinct levels of dietary $\omega$-3 LC-PUFA [12]. Several physiological processes were also previously attributed to the metabolites from $\omega$-3 LC-PUFA, especially DHA such as neuroprostane, or ALA such as phytoprostane, for metabolites from non-enzymatic oxidation [51-53] and resolvins for metabolites from enzymatic oxidation $[54,55]$. However, no investigations on their role and involvement in oro-gustatory perception in fishes have been done. Further studies will be necessary to elucidate the oxidation of $\omega$-3 LC-PUFA in the diet and in oro-sensory tissues of RT and the involvement of these metabolites in the regulation of feeding behavior of farmed fish, especially as natural ligand for taste receptors in the tongue and brain tissues.

For the other taste receptors detected, we found a highlevel expression of the $c d 36$ transcript in the oral cavity but no regulation by diets in the tongue. In mammals, CD36 is expressed in several tissues as observed in our study. CD36 is also known to have high affinity for FA [56], and to be expressed mainly in the circumvallate and fungiform papillae. To date there is evidence for the implication of CD36 in the oro-gustatory perception of dietary lipids in mammals [57]. The higher detection in the RT liver could be related to this function in lipid metabolism as observed in mammals. Compared to our results, the physiological function of CD36 could be strongly preserved between the species.

For other FA taste receptors, GPR84 is not implicated in oro-gustatory perception in mammals but plays an important role in immune and metabolic responses and may mediate the crosstalk between immune cells and adipocytes [58]. Our results support the same conclusion. Gene expression of $g \operatorname{pr} 119$ was mainly and highly expressed in the intestine and stomach of RT, suggesting the same physiological role reported in mammals, with regulation of metabolism, gastrointestinal tract, and insulin secretion [59]. No difference between diets was observed for the gene expression of gpr119 and gpr84. Hovewer, a recent study revealed the role of gpr119 and gpr84 in the central regulation of FI in RT ([27], p. 84). Additional studies will be required to determine the regulation of this receptor by nutrients, especially FA in tongue and brain tissues.

GNAT [guanine nucleotide-binding protein $\mathrm{g}(\mathrm{t})$ subunit alpha], also known as the gustducin gene, is a $\mathrm{G}$ alpha protein found in some taste receptor cells in mammals associated with the gustatory system. However, teleosts do not have an ortho$\log$ of the mammalian gustducin gene, which is gnat 3 (guanine nucleotide binding protein alpha transducing activity polypeptide 3). In our study, we found gene expression of gnat2 in the oral cavity but also in gill filaments with downregulation by the V1 diet in the tongue. GNAT2 is known to be expressed in rods and cones of mammals, and our results suggest the possible fish equivalent to mammalian gustducin. However further research has to be done because we cannot exclud this role by other protein-G alpha subunits, such as the GNAI family [guanine nucleotide-binding protein $\mathrm{G}(\mathrm{i})$ subunit alpha] and the GNAO family [guanine nucleotide-binding protein $\mathrm{G}(\mathrm{o})$ subunit alpha]. The high gene expression of gnat2, gpr84 and gpr119 in gill filaments could also suggest an FA sensing of this tissue in fishes. However, absence of research on this tissue requires further study to confirm the hypothesis.

As detection of nutrients remains poorly studied in fishes [24] we also investigated the detection and regulation of transcripts for the different amino acid taste receptors in mammals. In mammals, T1R receptors are involved in amino acid and carbohydrate sensing [60] in the tongue and have been detected in many extra-oral tissues and organs including the intestine [61]. In our study, the distribution of transcripts for the different amino acid T1R receptors were different between paralogs with higher mRNA level expression of tas $1 r 1$ paralogs in the oral cavity, higher expression of tas $1 r 2$ in the palate and pharynx and in the pharynx and liver for the tas $1 r 3$ transcript. Our findings revealed no regulation at the mRNA level of $\operatorname{tas} 1 r 2$ and tas $1 r 3$ by diets. Moreover, there was very little difference in the proportions of amino acids between the $\mathrm{C}$ and $\mathrm{V} 1$ diets and no regulation with the V2 diet (with the same amino acid composition and proportion as the V1 diet). This might explained the non-regulation of amino acid receptor in tongue tissue. Hovewer we can not exclude any involvement of $\mathrm{T} 1 \mathrm{R}$ receptors in the detection of nutrients in the oral cavity of RT. Further experiments are warranted with diets containing different amino acids profiles. The low level of gene expression of casr and gpr92/93 in the tongue supported the same conclusion as that for the T1R receptors.

\section{Conclusion}

In conclusion, this study for the first time deciphers the detection and modulation of taste receptors in RT fed a total plant based-diet supplemented or without $\omega-3$ LC-PUFA (DHA). The results of the present study show that RT have the fundamental mechanisms for oro-gustatory perception of nutrients related to different diet composition. In particular, we provide the first set of evidence suggesting that taste receptors like ffar 2 compared to ffar 1 and ffar4 in mammals were involved in oro-gustatory perception of FA sensing in $\mathrm{RT}$ and could play role in the regulation of feeding behavior. 
Further studies will be necessary to characterize the potential binding (agonist/antagonist) and role (sensing pathways) of taste receptors in the regulation of FA sensing in RT and to elucidate their implication in the regulation of feeding behavior. This knowledge is important in the aquaculture industry for diversification or substitution of feed ingredients, especially the already expensive and limited FM and other animal-based ingredients.

\section{Data Availability}

The data presented in this study are available on request from the corresponding author.

\section{Conflicts of Interest}

The authors declare that there are no conflicts of interests.

\section{Funding}

This study was supported by research grants from the INRAE PHASE Department (France).

\section{Supplementary Materials}

Supplemental information 1. Representation of experimental procedure for self-feeder experiment (20 days of acclimation period, followed by 30 days of feeding test and 10 days of preference test). Supplemental information 2. Selected amino acid diet composition (estimated, \% of dry matter). (Supplementary Materials)

\section{References}

[1] N. Pelletier, D. H. Klinger, N. A. Sims, J.-R. Yoshioka, and J. N. Kittinger, "Nutritional attributes, substitutability, scalability, and environmental intensity of an illustrative subset of current and future protein sources for aquaculture feeds: joint consideration of potential synergies and trade-offs," Environmental Science \& Technology, vol. 52, no. 10, pp. 5532-5544, 2018.

[2] D. M. Gatlin, F. T. Barrows, P. Brown et al., "Expanding the utilization of sustainable plant products in aquafeeds: a review," Aquaculture Research, vol. 38, no. 6, pp. 551-579, 2007.

[3] V. Lazzarotto, F. Médale, L. Larroquet, and G. Corraze, "Longterm dietary replacement of fishmeal and fish oil in diets for rainbow trout (Oncorhynchus mykiss): Effects on growth, whole body fatty acids and intestinal and hepatic gene expression," PloS One, vol. 13, no. 1, article e0190730, 2018.

[4] M. J. Leaver, L. A. Villeneuve, A. Obach et al., "Functional genomics reveals increases in cholesterol biosynthetic genes and highly unsaturated fatty acid biosynthesis after dietary substitution of fish oil with vegetable oils in Atlantic salmon (Salmo salar)," BMC Genomics, vol. 9, no. 1, p. 299, 2008.

[5] G. M. Turchini, B. E. Torstensen, and W.-K. Ng, "Fish oil replacement in finfish nutrition," Reviews in Aquaculture, vol. 1, no. 1, pp. 10-57, 2009.

[6] M. Sprague, J. R. Dick, and D. R. Tocher, "Impact of sustainable feeds on omega-3 long-chain fatty acid levels in farmed Atlantic salmon, 2006-2015," Scientific Reports, vol. 6, no. 1, 2016.
[7] B. E. Torstensen, M. Espe, M. Sanden et al., "Novel production of Atlantic salmon (Salmo salar) protein based on combined replacement of fish meal and fish oil with plant meal and vegetable oil blends," Aquaculture, vol. 285, no. 1, pp. 193-200, 2008.

[8] M. S. Izquierdo, "Essential fatty acid requirements of cultured marine fish larvae," Aquaculture Nutrition, vol. 2, no. 4, pp. 183-191, 1996.

[9] T. Benítez-Santana, E. Atalah, M. B. Betancor, M. J. Caballero, C. M. Hernández-Cruz, and M. Izquierdo, "DHA but not EPA, enhances sound induced escape behavior and Mauthner cells activity in Sparus aurata," Physiology \& Behavior, vol. 124, pp. 65-71, 2014.

[10] J. Roy, Y. Mercier, L. Tonnet et al., "Rainbow trout prefer diets rich in omega-3 long chain polyunsaturated fatty acids DHA and EPA," Physiology \& Behavior, vol. 213, 2020.

[11] J. Roy, L. Larroquet, A. Surget et al., "Impact on cerebral function in rainbow trout fed with plant based omega-3 long chain polyunsaturated fatty acids enriched with DHA and EPA," Fish \& Shellfish Immunology, vol. 103, pp. 409-420, 2020.

[12] J. Roy, C. Vigor, J. Vercauteren et al., "Characterization and modulation of brain lipids content of rainbow trout fed with $100 \%$ plant based diet rich in omega-3 long chain polyunsaturated fatty acids DHA and EPA," Biochimie, vol. 178, pp. 137-147, 2020.

[13] M. Behrens and W. Meyerhof, "Gustatory and extragustatory functions of mammalian taste receptors," Physiology \& Behavior, vol. 105, no. 1, pp. 4-13, 2011.

[14] N. Chaudhari and S. D. Roper, "The cell biology of taste," The Journal of Cell Biology, vol. 190, no. 3, pp. 285-296, 2010.

[15] M. Takeda, S. Sawano, M. Imaizumi, and T. Fushiki, "Preference for corn oil in olfactory-blocked mice in the conditioned place preference test and the two-bottle choice test," Life Sciences, vol. 69, no. 7, pp. 847-854, 2001.

[16] S. D. Roper and N. Chaudhari, "Taste buds: cells, signals and synapses," Nature Reviews. Neuroscience, vol. 18, no. 8, pp. 485-497, 2017.

[17] P. Besnard, P. Passilly-Degrace, and N. A. Khan, “Taste of fat: a sixth taste modality?," Physiological Reviews, vol. 96, no. 1, pp. 151-176, 2016.

[18] D. Gaillard and S. C. Kinnamon, "New evidence for fat as a primary taste quality," Acta Physiologica, vol. 226, no. 1, article e13246, 2019.

[19] C. A. Running, B. A. Craig, and R. D. Mattes, "Oleogustus: the unique taste of fat," Chemical Senses, vol. 40, no. 7, pp. 507$516,2015$.

[20] S. Abdoul-Azize, F. Atek-Mebarki, A. Bitam, H. Sadou, E. A. Koceïr, and N. A. Khan, "Oro-gustatory perception of dietary lipids and calcium signaling in taste bud cells are altered in nutritionally obesity-prone Psammomys obesus," PloS One, vol. 8, no. 8, article e68532, 2013.

[21] C. Cartoni, K. Yasumatsu, T. Ohkuri et al., "Taste preference for fatty acids is mediated by GPR40 and GPR120," The Journal of Neuroscience: The Official Journal of the Society for Neuroscience, vol. 30, no. 25, pp. 8376-8382, 2010.

[22] A. Sclafani, K. Ackroff, and N. A. Abumrad, "CD36 gene deletion reduces fat preference and intake but not post-oral fat conditioning in mice," American Journal of Physiology. Regulatory, Integrative and Comparative Physiology, vol. 293, no. 5, pp. R1823-R1832, 2007.

[23] S. Abdoul-Azize, S. Selvakumar, H. Sadou, P. Besnard, and N. A. Khan, " $\mathrm{Ca}^{2+}$ signaling in taste bud cells and spontaneous 
preference for fat: Unresolved roles of CD36 and GPR120," Biochimie, vol. 96, pp. 8-13, 2014.

[24] A. O. Kasumyan, "The taste system in fishes and the effects of environmental variables," Journal of Fish Biology, vol. 95, no. 1, pp. 155-178, 2019.

[25] F. Abbate, M. C. Guerrera, M. Levanti et al., "Anatomical, histological and immunohistochemical study of the tongue in the rainbow trout (Oncorhynchus mykiss)," Anatomia, Histologia, Embryologia, vol. 49, no. 6, pp. 848-858, 2020.

[26] A. S. Husted, M. Trauelsen, O. Rudenko, S. A. Hjorth, and T. W. Schwartz, "GPCR-mediated signaling of metabolites," Cell Metabolism, vol. 25, no. 4, pp. 777-796, 2017.

[27] C. Velasco, M. Conde-Sieira, S. Comesaña, M. Chivite, J. M. Míguez, and J. L. Soengas, "Role of the G protein-coupled receptors GPR84 and GPR119 in the central regulation of food intake in rainbow trout," Journal of Experimental Biology, vol. 242360, 2021.

[28] G. Nelson, J. Chandrashekar, M. A. Hoon et al., "An aminoacid taste receptor," Nature, vol. 416, no. 6877, pp. 199-202, 2002.

[29] I. Kimura, A. Ichimura, R. Ohue-Kitano, and M. Igarashi, "Free fatty acid receptors in health and disease," Physiological Reviews, vol. 100, no. 1, pp. 171-210, 2020.

[30] X.-C. Yuan, X.-F. Liang, W.-J. Cai, S. He, W.-J. Guo, and K.S. Mai, "Expansion of sweet taste receptor genes in grass carp (Ctenopharyngodon idellus) coincided with vegetarian adaptation," BMC Evolutionary Biology, vol. 20, no. 1, p. 25, 2020.

[31] The National Research Council [NRC], Nutrient Requirements of Fish and Shrimp, The National Academies Press, Washington, DC, 2011.

[32] V. Chromý, B. Vinklárková, L. Šprongl, and M. Bittová, “The Kjeldahl method as a primary reference procedure for total protein in certified reference materials used in clinical chemistry. I. a review of Kjeldahl methods adopted by laboratory medicine," Critical Reviews in Analytical Chemistry, vol. 45, no. 2, pp. 106-111, 2015.

[33] J. Folch, M. Lees, and G. H. Sloane Stanley, “A simple method for the isolation and purification of total lipides from animal tissues," The Journal of Biological Chemistry, vol. 226, no. 1, pp. 497-509, 1957.

[34] N. C. Shantha and R. G. Ackman, "Nervonic acid versus tricosanoic acid as internal standards in quantitative gas chromatographic analyses of fish oil longer-chain n-3 polyunsaturated fatty acid methyl esters," Journal of Chromatography, vol. 533, pp. 1-10, 1990.

[35] D. L. G. Noakes and J. F. Leatherland, "Social dominance and interrenal cell activity in rainbow trout, Salmo gairdneri (Pisces, Salmonidae)," Environmental Biology of Fishes, vol. 2, no. 2, pp. 131-136, 1977.

[36] T. Ellis, B. North, A. P. Scott, N. R. Bromage, M. Porter, and D. Gadd, "The relationships between stocking density and welfare in farmed rainbow trout," Journal of Fish Biology, vol. 61, no. 3, pp. 493-531, 2002.

[37] T. Boujard, L. Labbé, and B. Aupérin, "Feeding behaviour, energy expenditure and growth of rainbow trout in relation to stocking density and food accessibility," Aquaculture Research, vol. 33, no. 15, pp. 1233-1242, 2002.

[38] J. M. B. Grobler and C. M. Wood, "The physiology of rainbow trout in social hierarchies: two ways of looking at the same data," Journal of Comparative Physiology. B, Biochemical, Systemic, and Environmental Physiology, vol. 183, no. 6, pp. 787-799, 2013.
[39] J. Roy, F. Terrier, M. Marchand et al., "Effects of low stocking densities on Zootechnical parameters and physiological responses of rainbow trout (Oncorhynchus mykiss) juveniles," Biology, vol. 10, no. 10, p. 1040, 2021.

[40] T. Boujard, X. Dugy, D. Genner, C. Gosset, and G. Grig, "Description of a modular, low cost, eater meter for the study of feeding behavior and food preferences in fish," Physiology \& Behavior, vol. 52, no. 6, pp. 1101-1106, 1992.

[41] A. Kleiber, C. Valotaire, A. Patinote et al., "Rainbow trout discriminate 2-D photographs of conspecifics from distracting stimuli using an innovative operant conditioning device," Learning \& Behavior, vol. 49, no. 3, pp. 292-306, 2021.

[42] I. Geurden, A. Cuvier, E. Gondouin et al., "Rainbow trout can discriminate between feeds with different oil sources," Physiology \& Behavior, vol. 85, no. 2, pp. 107-114, 2005.

[43] S. Comesaña, M. Conde-Sieira, C. Velasco, J. L. Soengas, and S. Morais, "Oral and pre-absorptive sensing of amino acids relates to hypothalamic control of food intake in rainbow trout," The Journal of Experimental Biology, vol. 223, Part 17, 2020.

[44] M. W. Pfaffl, "A new mathematical model for relative quantification in real-time RT-PCR," Nucleic Acids Research, vol. 29, no. 9, article e45, pp. 45e-445, 2001.

[45] M. Nei, Y. Niimura, and M. Nozawa, "The evolution of animal chemosensory receptor gene repertoires: roles of chance and necessity," Nature Reviews. Genetics, vol. 9, no. 12, pp. 951$963,2008$.

[46] D. Shanmugamprema, K. Muthuswamy, G. Subramanian, V. Ponnusamy, V. Krishnan, and S. Subramaniam, "Fat taste signal transduction and its possible negative modulator components," Progress in Lipid Research, vol. 79, article 101035, 2020.

[47] J. B. Regard, I. T. Sato, and S. R. Coughlin, “Anatomical profiling of G protein-coupled receptor expression," Cell, vol. 135, no. 3, pp. 561-571, 2008.

[48] A. J. Brown, S. M. Goldsworthy, A. A. Barnes et al., "The Orphan G Protein-coupled Receptors GPR41 and GPR43 Are Activated by Propionate and Other Short Chain Carboxylic Acids*," The Journal of Biological Chemistry, vol. 278, no. 13, pp. 11312-11319, 2003.

[49] M. Grundmann, E. Bender, J. Schamberger, and F. Eitner, "Pharmacology of free fatty acid receptors and their allosteric modulators," International Journal of Molecular Sciences, vol. 22, no. 4, p. 1763, 2021.

[50] J.-M. Galano, J. Roy, T. Durand et al., "Biological activities of non-enzymatic oxygenated metabolites of polyunsaturated fatty acids (NEO-PUFAs) derived from EPA and DHA: new anti-arrhythmic compounds?," Molecular Aspects of Medicine, vol. 64, pp. 161-168, 2018.

[51] J. Roy, J. Fauconnier, C. Oger et al., "Non-enzymatic oxidized metabolite of DHA, 4(RS)-4- $\mathrm{F}_{4 \mathrm{t}}$-neuroprostane protects the heart against reperfusion injury," Free Radical Biology \& Medicine, vol. 102, pp. 229-239, 2017.

[52] J. Roy, J.-Y. Le Guennec, J.-M. Galano et al., "Non-enzymatic cyclic oxygenated metabolites of omega-3 polyunsaturated fatty acid: Bioactive drugs?," Biochimie, vol. 120, pp. 56-61, 2016.

[53] J. Roy, C. Oger, J. Thireau et al., "Nonenzymatic lipid mediators, neuroprostanes, exert the antiarrhythmic properties of docosahexaenoic acid," Free Radical Biology \& Medicine, vol. 86, pp. 269-278, 2015. 
[54] J. Roy, J.-M. Galano, T. Durand, J.-Y. Le Guennec, and J. C.-Y. Lee, "Physiological role of reactive oxygen species as promoters of natural defenses," FASEB Journal: Official Publication of the Federation of American Societies for Experimental Biology, vol. 31, no. 9, pp. 3729-3745, 2017.

[55] C. N. Serhan, S. Hong, K. Gronert et al., "Resolvins: a family of bioactive products of omega-3 fatty acid transformation circuits initiated by aspirin treatment that counter proinflammation signals," The Journal of Experimental Medicine, vol. 196, no. 8, pp. 1025-1037, 2002.

[56] A. G. Baillie, C. T. Coburn, and N. A. Abumrad, "Reversible binding of long-chain fatty acids to purified FAT, the adipose CD36 homolog," The Journal of Membrane Biology, vol. 153, no. 1 , pp. $75-81,1996$.

[57] F. Laugerette, P. Passilly-Degrace, B. Patris et al., "CD36 involvement in orosensory detection of dietary lipids, spontaneous fat preference, and digestive secretions," The Journal of Clinical Investigation, vol. 115, no. 11, pp. 3177-3184, 2005.

[58] H. Nagasaki, T. Kondo, M. Fuchigami et al., "Inflammatory changes in adipose tissue enhance expression of GPR84, a medium-chain fatty acid receptor: TNF $\alpha$ enhances GPR84 expression in adipocytes," FEBS Letters, vol. 586, no. 4, pp. 368-372, 2012.

[59] S. Odori, K. Hosoda, T. Tomita et al., "GPR119 expression in normal human tissues and islet cell tumors: evidence for its islet-gastrointestinal distribution, expression in pancreatic beta and alpha cells, and involvement in islet function," Metabolism: Clinical and Experimental, vol. 62, no. 1, pp. 7078, 2013

[60] C. Belloir, J. Savistchenko, F. Neiers, A. J. Taylor, S. McGrane, and L. Briand, "Biophysical and functional characterization of the N-terminal domain of the cat T1R1 umami taste receptor expressed in Escherichia coli," PloS One, vol. 12, no. 10, article e0187051, 2017.

[61] S. Steensels and I. Depoortere, "Chemoreceptors in the gut," Annual Review of Physiology, vol. 80, pp. 117-141, 2018. 